\title{
Niclosamide reverses SARS-CoV-2 control of lipophagy
}

Timothy J. Garrett ${ }^{1,2^{*}}$, Heather Coatsworth ${ }^{3 \ddagger}$, Iqbal Mahmud ${ }^{1,2 \ddagger}$, Timothy Hamerly ${ }^{3 \ddagger}$, Caroline J. Stephenson ${ }^{3,4}$, Hoda S. Yazd ${ }^{5}$, Jasmine Ayers ${ }^{3}$, Megan R. Miller ${ }^{3}$, John A. Lednicky ${ }^{3,4}$, Rhoel R. Dinglasan ${ }^{3^{*}}$

${ }^{1}$ Department of Pathology, Immunology, and Laboratory Medicine, College of Medicine, University of Florida, Gainesville, FL, 32610 USA

${ }^{2}$ Southeast Center for Integrated Metabolomics, Clinical and Translational Science Institute, University of Florida, Gainesville, FL, 32610 USA

${ }^{3}$ Emerging Pathogens Institute, Department of Infectious Diseases and Immunology, College of Veterinary Medicine, University of Florida, Gainesville, FL, 32611 USA

${ }^{4}$ Department of Environmental and Global Health, College of Public Health and Health Professions, University of Florida, Gainesville, FL, 32610 USA

${ }^{5}$ Department of Chemistry, University of Florida, Gainesville, FL 32603 USA

*Co-corresponding authors: tgarrett@ufl.edu and rdinglasan@epi.ufl.edu

${ }^{\ddagger}$ Authors contributed equally.

\section{SUMMARY}

2 The global effort to combat COVID-19 rapidly produced a shortlist of approved drugs with anti-

3 viral activities for clinical repurposing. However, the jump to clinical testing was lethal in some

4 cases as a full understanding of the mechanism of antiviral activity as opposed to pleiotropic 5 activity/toxicity for these drugs was lacking. Through parallel lipidomic and transcriptomic anal6 yses we observed massive reorganization of lipid profiles of infected Vero E6 cells, especially 7 plasmalogens that correlated with increased levels of virus replication. Niclosamide (NIC), a poorly soluble anti-helminth drug identified for repurposed treatment of COVID-19, reduced the 
9 total lipid profile that would otherwise amplify during virus infection. NIC treatment reduced the

10 abundance of plasmalogens, diacylglycerides, and ceramides, which are required for virus

11 production. Future screens of approved drugs may identify more druggable compounds than

12 NIC that can safely but effectively counter SARS-CoV-2 subversion of lipid metabolism thereby

13 reducing virus replication. However, these data support the consideration of niclosamide as a

14 potential COVID-19 therapeutic given its modulation of lipophagy leading to the reduction of

15 virus egress and the subsequent regulation of key lipid mediators of pathological inflammation.

17 Keywords: lipidomics, autophagy, lipophagy, SARS-CoV-2, coronavirus, COVID-19, mass

18 spectrometry, RNA sequencing, niclosamide, antivirals, metabolism, chemical biology

INTRODUCTION

The current pandemic spread of SARS-CoV-2 has resulted in $>600,000$ deaths in the United States alone and $>176 \mathrm{M}$ cases and $>3.8 \mathrm{M}$ deaths worldwide (Dong et al., 2020). In 2020, there was a rapid global effort to study severe acute respiratory syndrome coronavirus 2 (SARS-CoV-2) infection kinetics in vitro to identify pathways that are involved in entry, replication, and egress of the virus as new targets that can be treated with existing drugs (Gautret et al., 2020; Jeon et al., 2020; Liu et al., 2020; Vincent et al., 2005; Wang et al., 2020; Zhou et al., 2020). However, this worldwide effort was fraught with inconsistencies ranging from the mammalian host cell used for infection studies, to overall study design, which made it difficult to identify common host cell pathways critical to virus biology that can be targeted with available compounds.

Chloroquine $(\mathrm{CQ})$ and, hydroxychloroquine $(\mathrm{HCQ})$, which are indicated for treating ma- 
33 2020; Dyall et al., 2014; Liu et al., 2020; Mauthe et al., 2018; Vincent et al., 2005; Wang et al.,

34 2020). However, the direct and rapid translation of this finding to clinical studies during the

35 pandemic was either equivocal at best, or disastrous (Axfors et al., 2021). Although no studies

36 to date have specifically and comprehensively explored the underlying antiviral mechanism of

37 action (MOA) for these two drugs, they nonetheless moved quickly to COVID-19 clinical stud-

38 ies. $\mathrm{CQ}$ and $\mathrm{HCQ}$ are 4-aminoquinolines (4-AQ), and another human-safe 4-AQ drug,

39 Amodiaquine (AQ), has also been shown to have antiviral activity against SARS-CoV-1 and

40 Middle East respiratory syndrome coronavirus (MERS-CoV) (Dyall et al., 2014; Vincent et al.,

41 2005), as well as Ebola (D'Alessandro et al., 2020), suggesting that there are common MOAs

42 underpinning broad 4-AQ antiviral activity. Initially, these 4-AQ compounds were considered as

43 candidate partner drugs that can be used along with remdesivir (Wang et al., 2020), the lead-

44 ing broad-spectrum RNA synthesis inhibitor that has shown some clinical benefit (Goldman et

45 al., 2021). The exact nature of the cellular pathways and processes that are targeted by these

46 4-AQs resulting in reductions in virus propagation remain unknown, but direct protein interac-

47 tion studies with CQ suggest broad pleiotropic effects on the cell (Gordon et al., 2020). The

48 pleiotropic effects of drugs such as $C Q$ should have given pause, preventing its quick transla-

49 tion to the clinic wherein higher doses of this drug exacerbate the pleiotropic side effects that

50 can result in direct harm to the subject in clinical trials (Borba et al., 2020).

Another anti-parasitic drug, niclosamide (NIC), which suppresses MERS-CoV propaga-

52 tion by inhibiting autophagosome-lysosome fusion through the Beclin1 (Bec1, autophagy regu-

53 lator/antiapoptotic protein)-SKP2 (S-phase kinase-associated protein 2) pathway (Gassen et

54 al., 2019) has also been proposed to be a potent candidate drug for repurposing in the treat-

55 ment of SARS-CoV-2 (Xu et al., 2020). NIC is a salicylanilide, oral anti-helminthic drug used in 
56 human and veterinary medicine. Akin to the 4-AQs, it appears to have functional effects be-

57 yond the Bec1-SKP2 axis in a cell, resulting in broad anti-infective properties, and as a result,

58 also suffers from poor translational potential to the clinic for COVID-19. Considering the failure

59 of $\mathrm{CQ}$ and $\mathrm{HCQ}$ to progress towards clinical utility, the candidacy of NIC, which is at an earlier

60 stage of consideration for COVID-19 treatment, compelled us to examine more closely its

61 global effects on host cells to determine if we can uncouple the reported antiviral MOA via the

62 Bec1-SKP2 pathway as well as other putative antiviral mechanisms from intrinsic cytotoxicity.

63 We hypothesize that NIC exerts pleiotropic functional activities on a host cell that result in the

64 perturbation of two intimately associated cellular pathways: autophagy and lipid metabolism.

65 These two processes are dysregulated at critical SARS-CoV-2 life cycle checkpoints during virus

66 infection (Stukalov et al., 2021), and treatment with NIC is hypothesized to reverse this

67 dysregulation. Recently, a multi-omics analysis of clinical samples from COVID-19 patients also

68 described a marked change in lipidomics profiles that correlated with disease severity (Overmyer

69 et al., 2021), but this approach could not characterize the mechanism at the cellular level. Here-

70 in, we identified and characterized autophagic or lipophagic pathways (and wider linked cellular

71 networks) that are targeted by NIC in the presence and absence of a SARS-CoV-2 infection.

72 We used the lipid signatures induced by this anti-parasitic compound to detect pathways that

73 are predicted to either lead to direct antiviral effects or to cellular dysregulation and cell death.

74 We discuss the utility of this approach in informing the selection of repurposed drugs that may

75 have more clinical benefit, better bioavailability, and lesser cytotoxicity in the context of

76 COVID-19 and other infectious diseases. 


\section{SARS-CoV-2 infection alters the host cell lipidome}

The mechanism by which SARS-CoV-2 enters host cells and systematically alters the cellular environment is currently one of the most compelling areas of study to support the de-

82 velopment of antiviral interventions (V'kovski et al., 2020). Evidence suggests that SARS-CoV-

832 subverts pre-existing cellular lipids and lipid signaling mechanisms for entry, intracellular traf-

84 ficking and egress (Abu-Farha et al., 2020). This is consistent with what is known about virus-

85 es in general (Mazzon and Mercer, 2014). To investigate the role of lipids in SARS-CoV-2 dur-

86 ing the infection cycle in host cells, we profiled the global lipidome from Vero E6 cells at $16 \mathrm{~h}$

87 and $48 \mathrm{~h}$ post-infection with SARS-CoV-2 (Figure S1A). The correlation matrix across the 88 groups studied showed a distinct clustering in SARS-CoV-2 infected cells as well as clear clus-

89 tering with virus and NIC treatment (Figure S1B). In addition, a principal component analysis

90 (PCA) revealed clear clustering based on lipid profiles between early (16h) and late (48h)

91 timepoints post viral infection (Figure S1C). PCAs also showed clustering with NIC treatment

92 of virus infected cells at early (16h) and late timepoints (48h) (Figures S1D and E, respec-

93 tively). Measurement of supernatant SARS-CoV-2 genome copy with RT-qPCR revealed a

94 significantly higher quantity of released progeny genomes at $48 \mathrm{~h}$ than $16 \mathrm{~h}$, indicating that

95 more viral egress is occurring at the $48 \mathrm{~h}$ timepoint, which is consistent with literature (Figure

96 S2). We did not perform any measurements of infectious progeny virions (i.e., plaque assay),

97 so the antiviral effect of NIC in this experiment cannot be conclusively determined.

As SARS-CoV-2 infection caused perturbations in the global lipid profiles (Figure 2A), we next sought to characterize the modulations of different lipid classes between early (16h) vs

100 late (48h) timepoints post viral infection. In total, we identified 720 lipids from 34 classes (Ta101 ble S2, S3) in SARS-CoV-2 infected host cells covering all major lipid classes (Figure 2A). 
102 Phosphatidylcholine (PC), phosphatidylethanolamine (PE), plasmalogens (ether linked lipids) 103 and triacylglycerol (TG) represented the most frequent lipid classes identified, which is ex104 pected given their abundance in cell membranes and lipid droplets (Figure 2A, Figure 3). Hierarchical clustering analysis of the differentially regulated lipids (FC>1.5, adjusted p106 value $<0.05)$ revealed specific lipid classes were associated with early or late events during 107 productive virus infection (Figure 2A, Table S2). We found that ether lipids including 108 plasmanyl-TG, plasmenyl-TG, plasmanyl-LPC, plasmanyl-PC, plasmenyl-PC, and plasmenyl$109 \mathrm{PE}$, as well as diacylglycerides were elevated at 48h post viral infection (Figure 2B, Table 110 S3). We measured the relative abundance of the total ether linked lipids and observed a highly 111 significant elevation $(\mathrm{P}$-value $=2.94 \mathrm{E}-38)$ of total ether linked lipids at $48 \mathrm{~h}$ post viral infection 112 (Figure 2C, Figure S3), suggesting a potential role of ether linked lipids in SARS-CoV-2 path113 ogenesis. A comparative extracted ion chromatogram of plasmanyl-PC $(\mathrm{O}-18: 0 / 16: 0)+\mathrm{H}$, as an 114 example of a specific ether lipid is shown (Figure 2D) to convey the clear difference in profiles 115 between the two time points tested. On the other hand, TGs, cholesterol esters (CE), and 116 hexosylceramides (HexCer) were elevated at early stages of infection (16h) (Figure 2A and B,

117 Figure 3, Figure S3, Table S3). The repertoire of lipid classes that partitioned to early steps in 118 virus infection (16h) and later stages of increased virus replication and egress (48h) are shown 119 in Figure 3. Lipids that contained longer chain fatty acids with higher degrees of unsaturation includ121 ing TGs (4-9 double bonds (db)), PC (4-5 db), CL (5-6 db), PE (1-6 db), and PS (1-4 db) were 122 elevated in early (16h) viral infection (Figure 3, Table S3), a trend consistent with what was 123 described in SARS-CoV-2 infected golden Syrian hamsters (Rizvi et al., 2021). In contrast, 124 saturated fatty acids (SFA), monounsaturated fatty acids (MUFA) and long chain fatty acids 
125 (LCFA) were found to be significantly downregulated in early (16h) viral infection but elevated

126 at $48 \mathrm{~h}$ post viral infection (Table S3), consistent with previous reports (Koyuncu et al., 2013;

127 Nguyen et al., 2018). We identified changes in lipids that may be related to a cellular response

128 to compensate for virus energy utilization, viral particle formation from lipid droplets, vesicle 129 transport and autophagosome formation, noting a significant increase in plasmalogens (ether 130 lipids), sphingolipids (SM and CerNS), glycerophospholipids (PI, PS and PG), 131 lysophospholipids (LPE) and glycerolipids (DG) and a significant decrease in TG and CE lipids 132 at $48 \mathrm{~h}$ (Figure $2 \mathrm{~A}-\mathrm{B}$, Figure 3 ).

We observed a differential regulation of lipids during increased levels of viral replication.

134 Of note, we observed significant increases in PG, PS and PI, but not a significant change in 135 PC or PE phospholipids. This differential regulation of phospholipids could suggest formation 136 of membranes for the viral envelope as most mammalian cells are high in PC and PE phos137 pholipids (Deng and Angelova, 2021). The significant decrease in TGs and CEs is likely relat138 ed to virus utilization of lipid droplets to make viral particles needed for replication. Lipid drop139 lets are used by cells to store neutral lipids that are utilized for energy needs, but viruses re140 quire these lipids for replication and thus hijack the lipid droplets to enable their own growth. 141 The increase in plasmalogens is also associated with viral replication and increased levels 142 have been detected in the serum of patients with ZIKV and other viruses (Cloherty et al., 2020; 143 Mazzon and Mercer, 2014). 
To understand these lipid modulations in the context of therapeutic potential, we used

148 niclosamide (NIC), an anti-parasitic drug, which was found to suppress MERS-CoV propaga-

149 tion by enhancing autophagic flux through the Beclin1 (autophagy regulator/antiapoptotic pro-

150 tein)-SKP2 (S-phase kinase-associated protein 2) pathway (Gassen et al., 2019). NIC has also

151 been proposed to be a potent candidate drug for repurposing in the treatment of SARS and

152 COVID-19 (Gassen et al., 2021; Pindiprolu and Pindiprolu, 2020; Wu et al., 2004). The influ-

153 ence of SARS-CoV-2 infection on Vero E6 cell lipid metabolism, and inhibition of autophagic

154 flux encouraged us to assess if NIC may impart therapeutic potential to SARS-CoV-2 infection.

155 We first examined the effect of NIC on Vero E6 cells in the absence of infection to es-

156 tablish a baseline for both the 16h and 48h time points (Figure 4, Figure S4, Table S7). In to-

157 tal, we identified 520 lipids across all samples, with distinct profiles between DMSO control and

158 NIC treated cells. We observed a profound reduction across both time points in the lipid pro-

159 files for TG, and this effect was more pronounced at the $48 \mathrm{~h}$ time point (Figure 4A). In the ab-

160 sence of NIC treatment, plasmalogens (plasmanyl-PE, -TG, and -PC as well as plasmenyl-

161 LPC, -PE, -LPE, -PC and -TG) were found to be differentially abundant at 48h as compared to

162 16h, which is expected given the cell growth during this time frame (Figure 4A and Table S4).

163 However, at the $48 \mathrm{~h}$ time point ( $24 \mathrm{~h}$ post treatment) NIC reduced the abundance ( $>2$ log2 fold-

164 change) of HexCer-NDS, while in parallel, increased the abundance ( $>2$ log2 fold-change) of 165 plasmenyl- and plasmanyl-TG, PI, plasmanyl-PE and -PC, as well as BMPs (Figure 4B and 166 Figure S5).

Having established a baseline effect of NIC on lipid metabolism in Vero E6 cells, we

168 next sought to understand the effect of NIC on lipid metabolism during SARS-CoV-2 infection.

169 We globally profiled lipids from cells infected with SARS-CoV-2 and treated with DMSO as 
170 compared to cells infected with SARS-CoV-2 and treated with NIC at 16h (Figure S1B) and

171 48h (Figure 5A, Figure S1E) using our UHPLC-HRMS approach. A PCA identified a clear 172 separation between NIC treated and untreated SARS-CoV-2 infected samples (Figure S1B, D 173 and E). We observed that the treatment of Vero E6 cells with NIC for $24 \mathrm{~h}$ starting $24 \mathrm{~h}$ after 174 SARS-CoV-2 infection (48h) robustly impacted the global lipid profile as compared to NIC 175 treatment beginning simultaneously with SARS-CoV-2 infection (Figure 5B), suggesting that 176 the antiviral activity of NIC is also dependent on host cell state (in this case, infection as op177 posed to cell growth described above), which in turn correlated with higher levels of virus repli178 cation and virion production (Figure S2). However, no significant reduction in viral genome 179 copy was observed in (NIC) treated cells compared to DMSO controls at either timepoint (Fig180 ure S2). This corroborates previous findings that NIC does not act to inhibit virus entry or ge181 nome replication (Gassen et al., 2021; Pindiprolu and Pindiprolu, 2020). NIC reduced DG as 182 well as several plasmalogens, both of which would have otherwise been increased by SARS183 CoV-2 infection at the 48h time point (Figure 5A, Figure 2, Figure 3). Strikingly, we observed 184 that NIC treatment downregulated total ether lipids $(P=2.49 E-38)$ (Figure 5C), suggesting that 185 NIC may impart anti-viral effect via affecting ether lipid metabolism. As indicated earlier, we 186 observed an increase in ether lipids at 48h in cells infected with virus alone, suggesting a key 187 role of ether lipids in viral replication (Figure $2 \mathrm{C}$ ). Upon treatment with NIC, it appears that this 188 lipid pathway is disrupted significantly, and thus may be affecting viral replication.

190 Transcriptional profiling captures the broader cellular impact of virus infection and 191 treatment with niclosamide 
We assessed if virus-induced changes in lipidomic profiles corresponded to canonical

193 transcriptional regulation of genes that are known to be involved in lipid metabolism, autopha-

194 gy, phosphorylation, and vesicle transport. Furthermore, we explored if treatment with NIC al195 ters this profile, and whether time of NIC addition alters the course of infection. To do this, we 196 used RNASeq to capture the global cellular gene expression of i) Vero E6 cells over time (ef197 fect of time), ii) Vero E6 cells during cellular SARS-CoV-2 infection (effect of virus) and iii) fol198 lowing treatment with NIC (effect of drug).

We did not observe any significantly differentially expressed (DE) genes over time in our 200 Vero E6 culture alone (0h vs 16h, 0h vs 48h, 16h vs 48h) (Table S6), suggesting while the cel201 lular background of Vero E6 cells during these different time periods may be dynamic, the age 202 of the cell culture did not confound other comparisons made in this study. Similarly, we did not 203 observe any differences between cells treated with SARS-CoV-2 and NIC at 16h versus 48h. 204 We did, however, observe differences with NIC treated cells at 16h versus 48h (860 DE genes, 205474 up-regulated and 386 down-regulated at 48h) (Table S1B, Table S6), as well as SARS206 CoV-2 infected cells at 16h versus 48h (109 DE genes, 48 up-regulated and 61 down207 regulated at 48h) (Table S1B, Table S6).

SARS-CoV-2 infection resulted in significantly DE genes across all our comparison 209 groups (Table S1B, Table S5, Figure 6. Viral infection resulted in 585 DE genes at $16 \mathrm{~h}$ (474 210 up-regulated; 111 down-regulated), and 562 DE genes at 48h (433 up-regulated; 129 down211 regulated) (Table S5). SARS-CoV-2 and NIC conditions resulted in more DE genes than 212 SARS-CoV-2 infection alone; NIC and viral infection resulted in 3,083 DE genes (1,301 up213 regulated; 1,782 down-regulated) at 16h, and 1,595 DE genes (706 up-regulated, 889 down214 regulated) at $48 \mathrm{~h}$ (Table S5). Overrepresented GO terms associated in both up- and down- 
215 regulated DE datasets contained a large number of lipid phosphate metabolism, lipid transport, 216 autophagy and lysosome-associated terms. Terms related to phosphatidylinositol, 217 phosphatidylethanolamine and phosphatidylglycerol were especially prevalent, mirroring ob218 served changes in the lipidomics data. At 16h, viral infection induced an up-regulation of 219 GABARAPL1, MAP1LC3A, PIK3C3, USP30-ORG3a and TBK1 (also up-regulated at 48h), and 220 a down-regulation of STK11, LARP1, ZC3H12A, TFEB, TICAM1, GOLGA2, PIK3R2, IFI16, 221 ULK1, WDR81, SQSTM1 (also down-regulated at 48h), and HK2. As per viral infection, NIC 222 and virus treated cells (at 48h) also saw an up-regulation of GABARAPL1, MAP1LC3A, 223 USP30-ORG3a, BMF, and TBK1; WDR81 was also down-regulated in the NIC and virus 224 treatment at $48 \mathrm{~h}$.

There were significant transcriptomic differences noted at $16 \mathrm{~h}$ and $48 \mathrm{~h}$ with NIC treat-

226 ment (Figures 6E and F), and at 48h with NIC treatment and SARS-CoV-2 infection (Table 227 S1B, Table S7, Figure 6G. NIC treatment alone induced 2,447 DE genes at 16h (1,301 up228 regulated; 1,146 down-regulated), and 2846 genes at 48h (1,707 up-regulated; 1,139 down229 regulated), while viral infection and NIC treatment resulted in 3,617 DE genes at 16h $(1,698$ 230 up-regulated; 1,919 down-regulated), although no DE genes were noted by 48h (Figures 6E231 G, Table S7). Overrepresented GO terms with NIC treatment related to autophagosome as232 sembly, regulation of autophagy, intracellular lipid metabolism, lipid localization and lipid ho233 meostasis (Figure S6). Similar genes were noted for the effect of NIC as were with the effect 234 of virus. RB1CC1 was up-regulated at 16h with virus infection (Figure 6A) but was down235 regulated upon NIC treatment (Figure 6E) at 16h. Conversely, BMF was down-regulated with 236 NIC at 48h, but was up-regulated upon NIC and virus treatment at 48 hours. Other genes that 
237 were down-regulated with viral infection (GOLGA2, IFI16, WDR81, HK2, SQSTM1, ULK1) re-

238 mained down-regulated with NIC treatment (with and without virus).

\section{DISCUSSION}

A virus subjugates host lipid molecules as a vehicle for entry, intracellular transport, virus replication, assembly of infectious viral particles, and egress (Miyanari et al., 2007). Ether lipids have been identified as an important lipid class for efficient membrane trafficking, endocytosis, transcytosis, and internalization of particles (Thai et al., 2001; Deng and Angelova, 2021). Deficiency of ether lipids in several model systems affects plasma membrane function, as well as structural changes in the ER and Golgi cisternae (Bazill and Dexter, 1990; Gorgas et al., 2006). SARS-CoV-2 infection has demonstrated a diverse range of COVID-19 disease severities that correlate with increased viral replication (Fajnzylber et al., 2020). Unlike during the early infection stage at $16 \mathrm{~h}$, where virus load and replication is low, we observed that SARS-CoV-2 infection uniquely elevated ether linked lipids after $48 \mathrm{~h}$ of infection, a phase that represents increased virus replication, virus assembly and egress as well as host cell energy utilization (Abu-Farha et al., 2020; Dimitrov, 2004; Mazzon and Mercer, 2014). Increased viral 253 replication drives infection severity and may lead to a perturbation of the host cellular molecu254 lar landscape (Fajnzylber et al., 2020).

We observed that NIC treatment significantly downregulates the ether lipid profile during infection. We also observed that NIC treatment in Vero E6 cells causes a reduction in TG con-

257 tent at both $16 \mathrm{~h}$ and $48 \mathrm{~h}$ suggesting a disruption of lipid droplet formation. (Figure 4A). NIC 258 has previously been tested as an anti-obesity drug, and a study using it associated with a high 259 fat diet showed a decrease in total TGs (AI-Gareeb et al., 2017). NIC also affects several lipid 
260 species, including PE, which may stimulate the autophagy process and inhibit ether lipid eleva261 tion during viral propagation. However, NIC has dual activity in cells and is capable of influenc262 ing autophagic processes through canonical and non-canonical pathways (Liu, et al., 2019). 263 Whereas in uninfected cells, NIC reduces autophagic flux as well as the global lipid profile 264 (causing a decrease in plasmalogens, TGs and PE lipids), in infected cells, NIC induces au265 tophagy and the subsequent reduction of the ether lipid profile. NIC has been shown to affect 266 the function of lysosomes by preventing the acidification of the vacuole (Jung et al., 2019). 267 This disruption of lysosomal function results in increased autophagic flux similar to the likely 268 primary mechanism of action underlying the broad antiviral activity of chloroquine (Mauthe et 269 al., 2018). Importantly, we identified that ether linked lipids were only affected by NIC treat270 ment relative to productive virus infection at $48 \mathrm{~h}$, suggesting that the activity of NIC may be 271 dependent on the cellular state or level of virus burden and virus replication kinetics.

To understand the critical lipids related to autophagy activation, we analyzed the phosphatidylethanolamine (PE) levels in NIC treated SARS-CoV-2 infected cells. PE is one of

274 the central lipids that conjugates the factors for induction of autophagy through the formation of 275 the autophagosome (Xie et al., 2020). We observed that the relative abundance of PE was 276 significantly elevated with NIC treatment at $48 \mathrm{~h}$ while there was no impact of NIC on PE levels 277 at 16h, suggesting that NIC enhanced the autophagy machinery upon viral replication to direct278 ly affect downstream virus assembly, trafficking, and egress. Different signaling lipids such as 279 PS, PI, and PGs play critical roles in endosome trafficking and maturation (Mazzon and Mer280 cer, 2014) and help in infectious viral particle transport. Our study first identified that NIC 281 treatment significantly reduced the global level of different glycerophospholipids such as PS, $282 \mathrm{PI}$, and PG at 48h, but had no impact on early infection at $16 \mathrm{~h}$. 
TGs are the major form of lipids enriched in lipid droplets. Drugs that stimulate TG downregulation usually activate autophagy and may prevent a cell from viral infection and pathogenesis. We identified that NIC treatment significantly reduced global levels of TGs in SARS-CoV-2 at $48 \mathrm{~h}$ treatment, suggesting that NIC treatment potentiates TG breakdown in lipid droplets to help reduce viral replication. We also found that NIC treatment showed either no effect or very low impact on lipids that were involved in lipid droplet formation at $16 \mathrm{~h}$. In fasting conditions, lipid droplets are utilized for fatty acid oxidation to produce energy as intact TGs are broken down to release fatty acids in a process called lipophagy. The breakdown of 291 TGs via hydrolysis is mediated by autophagy and we observed a decrease in TGs with NIC 292 treatment. Overall, these findings demonstrate that NIC treatment likely activates autophagy 293 and consequently impacts ether linked lipids and other lipids needed for viral infection and propagation. extracellular vehicles (EVs), also act as cholesterol transporters in cooperation with other fac-

297 tors and facilitate viral infection and replication (Luquain-Costaz et al., 2020). Interestingly, our 298 study detected three different forms of BMP and two different forms of cholesterol esters (CE) 299 that were all significantly downregulated with NIC treatment. Besides the impact of NIC on dif300 ferent autophagy related lipids, we also investigated the role of NIC on bioactive lipid metabo301 lism and apoptosis. We observed a dichotomous pattern of CerNS and SM lipids, where 302 CerNS decreased after NIC treatment (48hV vs 48hV+NIC) while SM were increased, indica303 tive of cell death or cell survival, which may be partly explained by the cell state-dependency of 304 NIC that we have observed in the presence or absence of SARS-CoV-2 infection in Vero E6 305 cells. Interestingly, we identified a notable reduction of gangliosides detected as HexCer 
306 (which are also called glycosphingolipids) upon NIC treatment at 48h in both uninfected and

307 SARS-CoV-2 infected Vero E6. Gangliosides have been implicated in the induction of 308 autophagic death in mammalian cells (Hwang et al., 2010), suggesting that NIC can increase 309 cell survival at homeostasis as well as under stress/infection conditions. This activity may in 310 part contribute to the low cytotoxicity for this drug for several different cell lines, with the re311 ported $\mathrm{CC}_{50}=50 \mu \mathrm{M}$ (Jeon et al., 2020; Xu et al., 2020). Although gangliosides have also been 312 shown to bind cooperatively with ACE-2 to the SARS-CoV-2 spike protein (Fantini et al., 2021), 313 our experimental design did not include a pretreatment of cells prior to virus infection. The 314 search for highly safe drugs for prophylaxis against COVID-19 remains elusive and controver315 sial, but these data may suggest a role for niclosamide for future outbreaks given its broad an316 tiviral activities. Considering the potential link between plasmalogens and the observed cyto317 kine and lipid storms (Deng and Angelova, 2021) in severe COVID-19 patients, NIC may offer 318 to a two-pronged treatment approach, i.e., reduce virus abundance and provide a check on an 319 uncontrolled inflammatory response induced by SARS-CoV-2.

321 tured for each of the treatments/conditions, wherein hallmarks of and the induction of autopha322 gy related genes (such as DEPP1 (an autophagy regulator) (Salcher et al., 2017)), several li323 pases (Gassen et al., 2021), as well as genes implicated in the induction of apoptosis and 324 genes that are markers of cell proliferation. Furthermore, GO overrepresentation analyses 325 showed significantly upregulated terms such as death receptor activity, MAP kinase phospha326 tase activity, tumor necrosis factor-activated receptor activity, and phosphatidylinositol kinase 327 activity. To investigate the role NIC has on the reversal of SARS-CoV-2 infection induced au328 tophagy dysregulation, we explored the function of several genes that were differentially ex- 
pressed. RB1CC1 (RB1 inducible Coiled-Coil 1) along with ULK1 are part of the ULK complex

330 that initiates and regulates autophagy (Zachari and Ganley, 2017). RB1CC1 has also been

331 shown to influence viral infection (Mauthe and Reggiori, 2016), as a depletion of RB1CC1 led

332 to an increase of encephalomyocarditis virus replication. Here, RB1CC1 was up-regulated by

333 SARS-CoV-2 at 16h; however, was significantly down-regulated with treatment of NIC at $16 \mathrm{~h}$.

334 We also observed down-regulation of ULK1 in NIC treated cells at 16h. This elucidates the po-

335 tential role of RB1CC1 and ULK1 in early infection with SARS-CoV-2 and illuminates how NIC

336 may regulate autophagy. Interestingly, BMF was down-regulated at 48h with NIC, but up-

337 regulated upon NIC treatment of virus infected cells at 48h. BMF encodes for a Bcl-2-modifying

338 factor that is responsible for apoptotic regulation and has been reported as a target facilitating

339 viral evasion (Zamaraev et al., 2020). Additionally, autophagy genes IFI16 (Duan et al., 2011;

340 Jiang et al., 2021; Kim et al., 2020; Wichit et al., 2019), ZC3H12A (Srivastava et al., 2020),

341 SQSTM1 (Gassen et al., 2021), WDR81 (Zhu et al., 2021), and PIK3R2 (Weisberg et al.,

342 2020) are associated with viral infection, including SARS-CoV-1 (Gassen et al., 2021;

343 Srivastava et al., 2020) and were downregulated in this study when cells were treated with

344 NIC. Taken together, this provides evidence that SARS-CoV-2 infection leads to dysregulation

345 of autophagy, and that NIC acts to reverse this effect.

346 Several omics studies have made a significant contribution to the identification and un-

347 derstanding of potential antiviral therapies for COVID-19 (Mahmud and Garrett, 2020). Bioac-

348 tive molecules including PUFA lipids such as oleoylethanolamide (OEA), arachidonic acid

$349(\mathrm{AA})$, eicosapentaenoic acid (EPA), and docosahexaenoic acid (DHA) are known from existing

350 viral pathogenesis to inactivate enveloped viruses and inhibit pathogen proliferation/replication

351 (Das, 2020; Ghaffari et al., 2020; Tam et al., 2013). Accumulation of lipids including 
352 sphingolipids such as ceramides has been shown to negatively affect viral pathogenesis

353 (Soudani et al., 2019; Young et al., 2013). Lipid metabolism; specifically catabolism, biosyn-

354 thesis, and peroxidation play critical roles in autophagy or apoptosis-mediated cellular homeo355 stasis including cell survival and death (Xie et al., 2020). Autophagy can act as an anti-viral or 356 pro-viral mechanism; however, most viruses are found to inhibit autophagy signaling (Jackson, 357 2015). In the case of SARS-CoV-2, we know little about the relationship of virus infection with 358 autophagy signaling. Importantly, we identified elevated levels of ether lipids (plasmanyl and 359 plasmenyl) with increased viral replication, which was reported as a key lipid class for efficient 360 membrane trafficking (Thai et al., 2001). We revealed that lipids are critical molecular factors 361 for SAR-CoV-2 infection, entry, and viral replication into host cells. These lipids, which were 362 elevated by SARS-CoV-2 infection may be counteracted by PUFA and VLCFA that the virus 363 suppresses upon infection and entry (Kang et al., 2019).

371 ether lipids, TGs, and other lipid molecules. Reduction of these key lipids by NIC treatment 372 may lead to inhibition of SARS-CoV-2-induced signaling, which in turn drives viral endocytosis, 373 vesicular trafficking, propagation, and viral egress. Activating autophagy using small molecule 374 inhibitors/activators, oxysterols, or peptides is a promising approach for treating viral infections 
375 (Rubinsztein et al., 2012). However, most of the potential treatments (including NIC) suffer

376 translational obstacles due to adverse side effects, inefficient drug delivery to target tissues,

377 and poor bioavailability (Skotland et al., 2015). Although NIC is considered an essential medi-

378 cine and is well tolerated, the long history of dosing safety is based on per os delivery and anti-

379 parasitic activity within the gastrointestinal tract. SARS-CoV-2 infection begins in the lungs but

380 has been shown in several clinical studies to have a cosmopolitan infectivity profile, including

381 the brain, heart, and gastrointestinal tract. Notably, several repurposed drugs for COVID-19

382 were found to be associated with autophagy induction (Kim et al., 2012), raising the possibility

383 that some drugs that are already in clinical use for the treatment of parasitic or bacterial infec-

384 tions may be acting, at least in part, via autophagy. The recent clinical testing of intranasal or

385 inhaled NIC (Backer et al., 2021) suggests that the potential of this drug for use in mitigating

386 COVID-19 severity is profound, compeling expansive exploration of different approaches to 387 increase targeted delivery and efficacy.

\section{Limitations}

Although we used a C. sabaeus kidney epithelial cell line (Vero E6) and a SARS-CoV-2

391 strain isolated from a Floridian patient, we observed a similar (in direction and magnitude)

392 transcriptomic response to other SARS-CoV-2 transcriptomic studies, despite differences in

$393 \mathrm{MOI}$ and experimental sampling timepoints. These changes were consistent across another

394 Vero E6 study noting cell stress and apoptosis (DeDiego et al., 2011), as well as other cell sys-

395 tems, including human lung cells (Wyler et al., 2021), cardiomyocytes (Sharma et al., 2020),

396 adenocarcinomic human alveolar basal epithelial cells (Daamen et al., 2021), and bronchial

397 epithelial cells (Yoshikawa et al., 2010), infected with different betacoronaviruses, suggesting 
398 the responses detected herein may represent a core set of host SARS-CoV viral response 399 genes, and that these genes are not exclusive to our study system. Our work is limited to an in 400 vitro model; however, it was recently shown in a multiomics study of COVID-19 patient sam401 ples that the lipidomic profile can effectively partition COVID-19 disease severity (Overmyer et 402 al., 2021); implying that the biology captured in our culture model is relevant in vivo. While 403 these observations highlight potential utility of niclosamide, these data alone do not support its 404 indication in the clinic in the treatment of COVID-19.

406 STAR METHODS

407 RESOURCE AVAILABILITY

408 Lead Contact

409 Further information and requests for resources and reagents should be directed to and will be

410 fulfilled by the lead contact, Dr. Timothy Garrett (tgarrett@ufl.edu).

\section{Materials availability}

412 This study did not generate new unique reagents.

\section{Data and code availability}

414 The raw and processed data generated here have been deposited in publicly accessible data-

415 bases; the RNA sequencing data is available through NCBl's GEO repository (Accession:

416 GSE178157), and the lipidomics data is accessible via MetaboLights (Accession:

417 MTBLS2943).

418 This paper does not report original code. 
419 Any additional information required to reanalyze the data reported in this paper is available

420 from the lead contact upon request.

\section{EXPERIMENTAL MODEL AND SUBJECT DETAILS}

\section{Vero E6 cells.}

423 Vero clone E6 cells (ATCC: CRL-1586) were obtained from Dr. Pei-Yong Shi (University of

424 Texas Medical Branch). Vero E6 cells are an immortalized kidney epithelial cell line obtained 425 from the African green monkey (Cercopithecus sabeus). The cells are female.

426 Cells were maintained at $37^{\circ} \mathrm{C}$ and $5 \% \mathrm{CO}_{2}$, in Dulbecco's Modified Eagle Medium

427 (ThermoFisher \#11965118) supplemented with 10\% heat-inactivated fetal bovine serum 428 (FBS), 1X L-glutamine (Gibco \#25030-081), and 1X Penicillin/Streptomycin (Corning \#30-001$429 \mathrm{Cl})$. These cells have been authenticated.

430 SARS-CoV-2.

431 SARS-CoV-2/human/USA/UF-13/2020 (GenBank: MT620766.1) was passaged in Vero E6 432 cells, with culture conditions as described above except using reduced serum media with $3 \%$ 433 FBS rather than 10\% FBS. Virus was collected after 4 days to establish a low passage virus 434 stock within our biosafety level 3 (BSL-3) laboratory at the Emerging Pathogens Institute.

\section{METHOD DETAILS}

436 Cell culture, infection, and niclosamide treatment.

437 Cells were seeded in 6 well plates at a density of 500,000 cells/well. Each replicate sample 438 represents an individual plate well, with three biological replicates per condition, per time point. 439 The investigators were not blinded to the conditions. At hour zero (48 hours post seeding), 440 media was removed and replaced with reduced serum Dulbecco's Modified Eagle Medium 
441 (supplemented with 3\% FBS, 1X L-glutamine, and 1X Penicillin/Streptomycin) to facilitate in442 fection.

One vial of SARS-CoV-2 stock was thawed and diluted to a titer of $2 \times 10^{7}$ plaque form444 ing units (PFU) per mL. For infected conditions, virus was inoculated into each well with 12.5 $445 \mu \mathrm{L}$ of diluted virus stock for a multiplicity of infection (MOI) of 0.5 infectious virus particles/ Vero 446 E6 cell. Niclosamide (MedChemExpress \#HY-B0497, [NIC]) stock was dissolved in molecular 447 grade DMSO and added to the indicated wells for a final concentration of $5 \mu \mathrm{M}(0.5 \% \mathrm{v} / \mathrm{v}$ 448 DMSO final concentration). An equivalent amount of molecular grade DMSO was used as a 449 vehicle control. This concentration of NIC was selected to have antiviral effect but be well be450 low the observed $50 \%$ cytotoxic concentration $\left(\mathrm{CC}_{50}\right)$ of the drug $(50 \mu \mathrm{M})$ to avoid inadvertent 451 cell lysis in treated conditions (Wu et al., 2004). 454 collected $48 \mathrm{~h}$ after cell seeding. The "16 hour" (16h) conditions were infected with virus, treat455 ed with NIC or DMSO at hour zero, and harvested at hour 16 of the experiment (Figure 1). 456 The "48 hour" (48h) conditions were infected with virus at hour zero, supernatant was removed 457 after $24 \mathrm{~h}$, and cells were treated with NIC or DMSO in fresh media. Cells were harvested at 458 hour 48. Uninfected samples (with and without drug treatment) were also prepared and col459 lected as above. For samples from infected conditions, the supernatant was removed at the 460 indicated harvest timepoint, taking care not to disturb the cell monolayer, and retained at $46180^{\circ} \mathrm{C}$. The cells were washed once with $1 \mathrm{X}$ PBS. The PBS wash was removed and $0.25 \%$ 462 trypsin-EDTA (ThermoFisher \#25200056) was added to each well. The plates were then incu463 bated at $37^{\circ} \mathrm{C}$ and $5 \% \mathrm{CO}_{2}$ for $5-10$ minutes until cells had completely detached from the cell 
464 surface. Trypsinization was halted by the addition of $1 \mathrm{~mL}$ FBS. The supernatant was re465 moved, the cell pellet was resuspended in 1X PBS, and the sample was centrifuged at 700 466 RPM for 5 minutes. Supernatant was aspirated, and 100\% methanol was added to each sam467 ple purposed for lipid analysis. Alternatively, $750 \mu \mathrm{L}$ of TRIzol reagent (ThermoFisher 468 \#15596026) was added to each sample purposed for RNASeq. All samples were stored at $46980^{\circ} \mathrm{C}$ until analysis. For uninfected conditions, harvest proceeded the same as described 470 above except trypsinization was halted using $4 \mathrm{~mL}$ of complete Dulbecco's Modified Eagle 471 Medium instead of FBS, and pellets were flash frozen in liquid nitrogen instead of being resus472 pended in a chemical inactivation agent after the last centrifugation step. All samples were 473 stored at $-80^{\circ} \mathrm{C}$ until analysis.

\section{Global lipid extraction.}

475 Cell pellets from each time point and condition were extracted using a modified Folch biphasic extraction procedure (Ulmer, et al. 2017). Samples were pre-normalized to the protein concen-

477 tration $(800 \mu \mathrm{g} / \mathrm{mL})$ obtained using a Qubit 4.0 Fluorometer (Thermo Fisher Scientific). Lipids 478 were extracted using ice cold 4:2:1 chloroform:methanol:water (v:v:v) containing $20 \mu \mathrm{L}$ of a 479 10X diluted internal standard mixture (stock solution of $50 \mathrm{ppm}, \mathrm{w}$ : v), and the organic phase 480 was collected after centrifugation at $3260 \mathrm{xg}$ for $5 \mathrm{~min}$ at $4^{\circ} \mathrm{C}$, dried under nitrogen gas, and 481 reconstituted in $75 \mu \mathrm{L}$ of Isopropanol (IPA) plus $1 \mu \mathrm{L}$ of injection standard mixture (100 ppm, 482 w:v). Internal standards used in this analysis covered a range of lipid classes and structures 483 including: lysophosphatidylcholine (LPC 17:0), phosphatidylcholine (PC 17:0/17:0), 484 phosphatidylethanolamine (PE 15:0/15:0), phosphatidylserine (PS 14:0/14:0), 485 phosphatidylglycerol (PG 14:0/14:0), ceramide (Cer d18:1/17:0), diacylglycerol (DG 14:0/14:0), 486 and sphingomyelin (SM d18:1/7:0). For injection standards, triacylglycerol (TG 17:0/17:0/17:0), 
LPC 19:0, PE 17:0/17:0, PS 17:0/17:0, and PG 17:0/17:0 were used. Except for TG, all other

488 lipid standards were purchased from Avanti Polar Lipids (Alabaster, AL) while TG was pur489 chased from Sigma Aldrich. All lipid standards were diluted prior to analysis in 1:2 (v/v) chloro490 form:methanol $\left(\mathrm{CHCl}_{3}: \mathrm{MeOH}\right)$ and a working standard mix was then prepared by diluting the 491 stock solution with the same solvent mixture.

492 LC-MS-based lipid data collection and analysis.

493 Ultra-high-pressure liquid chromatography coupled to high resolution mass spectrometry 494 (UHPLC-HRMS) was used for data collection. Chromatographic separation was achieved us495 ing reversed phase chromatography (Dionex Ultimate 3000 RS UHLPC system, Thermo Sci496 entific) with a Waters Acquity C18 BEH column $(2.1 \times 50 \mathrm{~mm}, 1.7 \mu \mathrm{m})$ (Waters, Milford, MA, 497 US). The mobile phases consisted of solvent A (60:40 acetonitrile:water) and solvent B 498 (90:8:2) isopropanol:acetonitrile:water), both containing $10 \mathrm{mM}$ ammonium formate and $0.1 \%$ 499 formic acid. The flow rate was $500 \mu \mathrm{L} / \mathrm{min}$ and the column temperature was maintained at $50050^{\circ} \mathrm{C}$. A multi-step gradient was used for separation starting with $0 \% \mathrm{~B}$ from $0-1 \mathrm{~min}$, increas501 ing to $30 \%$ B from $1-3 \mathrm{~min}$, then up to $45 \%$ B from 3-4 min, $60 \%$ B from $4-6 \mathrm{~min}, 65 \%$ B from 502 6-8 min, held at 65\% B from 8-10 min, increased to $90 \%$ B from 10-15 min, then increased to $50398 \%$ B from $15-17$ min and finally held at $98 \%$ B from $17-18$ min before returning to initial con504 ditions to equilibrate for the next injection. Samples were analyzed in positive and negative 505 electrospray ionization as separate injections on a ThermoScientific Q-Exactive high resolution 506 mass spectrometer (Thermo Scientific, San Jose, CA). Lipidomics data were compiled and an507 notated using LipidMatch (Koelmel et al., 2017). 


\section{RNA extraction.}

509 For total RNA extraction from flash frozen cells, the cell pellet was thawed on ice. Cell pellets 510 were resuspended in $1 \mathrm{~mL}$ Trizol, and $200 \mu \mathrm{L}$ chloroform was added to each sample. Samples 511 were mixed thoroughly by vortexing, then incubated at room temperature for 3 minutes. Sam512 ples were centrifuged at $12,000 \times g$ for 15 minutes at $4^{\circ} \mathrm{C}$. The upper aqueous layer was trans513 ferred to a new tube and $500 \mu \mathrm{L}$ isopropanol was added and mixed by vortexing, prior to incu514 bation at room temperature for 10 minutes. RNA was pelleted by centrifuging at $12,000 \mathrm{xg}$ for 51510 minutes at $4^{\circ} \mathrm{C}$, and supernatant was discarded. The pellet was rinsed in $75 \%$ ethanol and pelleted by centrifuging at $7,500 \mathrm{xg}$ for 5 minutes at $4^{\circ} \mathrm{C}$. The ethanol wash and spin were re-

517 peated a second time. The pellet was air dried in an inverted tube. Genomic DNA was digest518 ed using the TURBO DNA-free ${ }^{\text {TM }}$ Kit (Invitrogen \#AM1907) according to manufacturer instruc519 tions. Samples were mixed with $350 \mu \mathrm{L}$ buffer RLT from the RNeasy®) Mini kit (Qiagen 520 \#74104) and $250 \mu \mathrm{L}$ 100\% ethanol, then transferred to a RNeasy® Mini kit spin column. Col521 umns were centrifuged at 8,000 xg for 15 seconds at room temperature, and flow-through was 522 discarded. Buffer RPE $(500 \mu \mathrm{L})$ was added to the column, which was centrifuged at the same 523 conditions, and flow-through was discarded. The RPE wash was repeated a second time. The 524 column was transferred to a fresh collection tube and centrifuged at $12,000 \times g$ for 1 min to dry. 525 RNA was eluted by adding $50 \mu \mathrm{L}$ of nuclease free water and centrifuging for 1 minute at 8,000 $526 \mathrm{xg}$ and was immediately frozen at $-80^{\circ} \mathrm{C}$. To extract viral RNA from culture supernatant, super527 natant was thawed, $200 \mu \mathrm{L}$ of supernatant was mixed with $200 \mu \mathrm{L}$ DNA/RNA Shield, and RNA 528 was immediately extracted using the Quick-DNA/RNATM Viral MagBead kit (Zymo Research 529 \#R2140), according to manufacturer instructions and then immediately frozen at $-80^{\circ} \mathrm{C}$. 


\section{Real-time RT-qPCR.}

531 To quantify viral genome copy in supernatant, one step real time reverse transcription qPCR

532 (RT-qPCR) was performed using 4x Quantabio UltraPlex 1-Step ToughMix No Rox (VWR 533 \#10804-946) and CDC 2019-nCoV_N1 (nucleocapsid) primer and probe mix (see Key Re-

534 sources Table for primer and probe sequences) at a final concentration of $22.5 \mu \mathrm{M}$, in a $20 \mu \mathrm{L}$ 535 reaction volume with $5 \mu \mathrm{L}$ template. All samples were run in technical duplicates. RT-qPCR 536 was run on a BioRad CFX96 ${ }^{\mathrm{TM}}$ Real-Time System. The thermal cycling conditions were as fol537 lows: $50^{\circ} \mathrm{C}$ for 20 minutes, $94^{\circ} \mathrm{C}$ for $2 \mathrm{~min}$; followed by 45 cycles of $94^{\circ} \mathrm{C}$ for 15 seconds, $55^{\circ} \mathrm{C}$ 538 for 30 seconds, and $68^{\circ} \mathrm{C}$ for 10 seconds. The samples were quantified using a standard curve 539 generated using a 2019-nCoV_N_Positive Control plasmid (IDT \# 10006625). Standard curve 540 points were plotted in Microsoft Excel v.2102 and cycle threshold values of unknown samples 541 were determined from the logarithmic line of best fit equation to calculate genome copies $/ \mathrm{mL}$.

\section{RNASeq analysis.}

543 All 27 RNA samples (see Table S1A-B) were directly sent to Novogene 544 (https://en.novogene.com/), where in-house RNA quality control and library preparation was 545 performed. Libraries were sequenced on an Illumina NovaSeq6000 platform using a 150bp kit 546 with paired-end read mode.

547 Mapping, expression, and pathway analyses.

548 Bioinformatic processing was completed using Galaxy (https://galaxyproject.org, (Batut et al., 549 2018)). Quality control was performed with Cutadapt v 1.16 (Martin, 2011) and FastQC v $550 \quad 0.11 .8$ (http://www.bioinformatics.babraham.ac.uk/projects/fastqc/) to remove adapters, <20 551 nucleotide reads and low-quality reads. RNA STAR v2.7.7a (Dobin et al., 2013) was used to 552 map samples to the Chlorocebus sabaeus genome and associated annotation (GenBank ac- 
553 cession \# GCA_015252025.1). featureCounts v 2.0.1 (Liao et al., 2014) was used with Infer

554 Experiment v2.6.4.1 (Wang et al., 2012) to determine the strandedness of the samples, and

555 sum reads for each gene. Read counts for each sample were input into DESeq2 v 1.22 .1

556 (Love et al., 2014) to call differential gene expression, analyzing the effect of time, drug, and

557 virus on the samples. Resultant differential expression files were manually annotated using a

558 combination of NCBI (O'Leary et al., 2016) and Ensembl (Yates et al., 2020) due to the poor

559 annotation quality of the genome

560 (https://www.ncbi.nlm.nih.gov/genome/annotation_euk/Chlorocebus_sabaeus/100/). Signifi-

561 cantly regulated gene names were separately parsed out for each comparison and uploaded to

562 ENRICHR (Kuleshov et al., 2016) for downstream gene ontology (GO (Harris et al., 2004)) and 563 pathway analyses using Reactome (Fabregat et al., 2016), MSigDB (Liberzon et al., 2015), 564 and KEGG (Ogata et al., 1999)) databases.

\section{QUANTIFICATION AND STATISTICAL ANALYSIS}

566 Unless indicated in the figure legend, all experiments were performed in triplicate and results 567 are presented as mean \pm standard error of mean (SEM) of absolute values or percentages of 568 control. Each replicate was a separate tissue culture well processed in parallel. A list of all 569 samples used for subsequent analysis can be seen in Table S1A-B.

570 Statistical P-values were obtained by application of the appropriate statistical tests using 571 GraphPad Prism v.9.0. Lipidomics data was normalized to the total ion signal, glog trans572 formed, autoscaled and analyzed with Metaboanalyst v.3.0 (Pang et al., 2021). For quality con573 trol, internal lipid standards added to each sample were used to assess technical reproducibil574 ity, achieving $\leq 15 \%$ relative standard deviation (RSD) across all samples. Correlation 
575 heatmaps were generated for the lipid samples using Pearson's r. Heatmap clustering was 576 performed using a T-test or an ANOVA, showing the most significant lipids. For lipidomics ap-

577 plications, Bonferroni false discovery rate (FDR) adjusted P-values lower than 0.05 and an ab578 solute fold-change greater than or equal to 1.5 were considered significant. For RNASeq dif579 ferential gene expression, gene ontology and pathway analyses, statistical significance was 580 corrected for multiple comparisons (Bonferroni adjusted) and assessed at an $\alpha=0.05$. Genes 581 with absolute $\log _{2}$ fold changes $\geq 1$ were considered significantly differentially expressed. For 582 supernatant genome copy RT-qPCR, a two-way non-parametric ANOVA with Dunn's post-hoc 583 test was performed in GraphPad Prism v.6.0 and assessed at an $\alpha=0.05$. Since no statistical 584 differences were observed between technical replicates of the same condition in the superna585 tant genome copy RT-qPCR data, technical replicate wells were pooled in the reported analy586 sis.

\section{$587 \quad$ AUTHOR INFORMATION}

\section{Corresponding Author}

589 Contact information for the author(s) to whom correspondence should be addressed:

590 Rhoel R. Dinglasan rdinglasan@epi.ufl.edu and Timothy J. Garrett tgarrett@ufl.edu

\section{Acknowledgements}

593 This work was funded in part by support from the University of Florida Preeminence Initiative 594 through the College of Veterinary Medicine and the Emerging Pathogens Institute (RRD).

\section{Author Contributions}


597 The manuscript was written through contributions by all authors. RRD, TG and JAL conceptu598 alized the study. IM, HC, TH, CJS, and JA conducted the study and generated samples and 599 data. IM, HC, TH, MM, JA, HSY, RRD and TG analyzed the data. IM, HC, TH, MM, JA, JAL, 600 RRD and TG wrote/edited the manuscript. All authors have given approval to the final version 601 of the manuscript.

$602 \ddagger$ These authors contributed equally.

603 Declaration of Interests

604 The authors declare no competing interests.

605 REFERENCES

606 Abu-Farha, M., Thanaraj, T.A., Qaddoumi, M.G., Hashem, A., Abubaker, J., and Al-Mulla, F. 607 (2020). The Role of Lipid Metabolism in COVID-19 Virus Infection and as a Drug Target. Int. J. 608 Mol. Sci. 21.

609 Al-Gareeb Al, Aljubory KD, Alkuraishy HM. Niclosamide as an anti-obesity drug: an experi610 mental study. Eat Weight Disord. 2017 Jun;22(2):339-344. doi: 10.1007/s40519-017-0373-1. 611 Epub 2017 Mar 7. PMID: 28271456.

612 Axfors, C., Schmitt, A.M., Janiaud, P., Van't Hooft, J., Abd-Elsalam, S., Abdo, E.F., Abella, 613 B.S., Akram, J., Amaravadi, R.K., Angus, D.C., et al. (2021). Mortality outcomes with 614 hydroxychloroquine and chloroquine in COVID-19 from an international collaborative meta615 analysis of randomized trials. Nat. Commun. 12, 2349.

616 Backer V, Sjöbring U, Sonne J, Weiss A, Hostrup M, Johansen HK, Becker V, Sonne DP, 617 Balchen T, Jellingsø M, Sommer MOA. (2021) A randomized, double-blind, placebo-controlled 618 phase 1 trial of inhaled and intranasal niclosamide: A broad spectrum antiviral candidate for 619 treatment of COVID-19. Lancet Reg Health Eur. 4, 100084. 
620 Batut, B., Hiltemann, S., Bagnacani, A., Baker, D., Bhardwaj, V., Blank, C., Bretaudeau, A., 621 Brillet-Guéguen, L., Čech, M., Chilton, J., et al. (2018). Community-Driven Data Analysis Train622 ing for Biology. Cell Syst. 6, 752-758.e1.

623 Bazill, G.W., and Dexter, T.M. (1990). Role of endocytosis in the action of ether lipids on 624 WEHI-3B, HL60, and FDCP-mix A4 cells. Cancer Res. 50, 7505-7512.

625 Borba, M.G.S., Val, F.F.A., Sampaio, V.S., Alexandre, M.A.A., Melo, G.C., Brito, M., Mourão, 626 M.P.G., Brito-Sousa, J.D., Baía-da-Silva, D., Guerra, M.V.F., et al. (2020). Effect of High vs 627 Low Doses of Chloroquine Diphosphate as Adjunctive Therapy for Patients Hospitalized With 628 Severe Acute Respiratory Syndrome Coronavirus 2 (SARS-CoV-2) Infection: A Randomized 629 Clinical Trial. JAMA Netw. Open 3, e208857.

630 Braga, L., Ali, H., Secco, I., Chiavacci, E., Neves, G., Goldhill, D., Penn, R., Jimenez631 Guardeño, J.M., Ortega-Prieto, A.M., Bussani, R., et al. (2021) Drugs that inhibit TMEM16 pro632 teins block SARS-CoV-2 spike-induced syncytia. Nature 594, 88-93.

633 Di Cara, F., Maile, T.M., Parsons, B.D., Magico, A., Basu, S., Tapon, N., and King-Jones, K. 634 (2015). The Hippo pathway promotes cell survival in response to chemical stress. Cell Death 635 Differ. 22, 1526-1539.

636 D’Alessandro, S., Scaccabarozzi, D., Signorini, L., Perego, F., Ilboudo, D.P., Ferrante, P., and 637 Delbue, S. (2020). The Use of Antimalarial Drugs against Viral Infection. Microorganisms 8.

638 Daamen, A.R., Bachali, P., Owen, K.A., Kingsmore, K.M., Hubbard, E.L., Labonte, A.C., Robl, 639 R., Shrotri, S., Grammer, A.C., and Lipsky, P.E. (2021). Comprehensive transcriptomic analy640 sis of COVID-19 blood, lung, and airway. Sci Rep. 11, 7052.

641 Daelemans, D., Pauwels, R., De Clercq, E., and Pannecouque, C. (2011). A time-of-drug addi642 tion approach to target identification of antiviral compounds. Nat. Protoc. 6, 925-933.

643 Das, U.N. (2020). Can Bioactive Lipids Inactivate Coronavirus (COVID-19)? Arch Med Res 51, 644 282-286.

645 DeDiego, M.L., Nieto-Torres, J. L., Jiménez-Guardeño, Regla-Nava, J.A., Alvarez, E., 646 Oliveros, J.C., Zhao, J., Fett, C., Perlman, S., and Enjuanes, L. (2011). Severe acute respirato- 
647 ry syndrome coronavirus envelope protein regulates cell stress response and apoptosis. PLoS 648 Pathog. 7, e1002315.

649 Deng, Y., and Angelova, A. (2021). Coronavirus-Induced Host Cubic Membranes and Lipid650 Related Antiviral Therapies: A Focus on Bioactive Plasmalogens. Front. Cell Dev. Biol. 9, 651630242.

652 Dimitrov, D.S. (2004). Virus entry: molecular mechanisms and biomedical applications. Nat. 653 Rev. Microbiol. 2, 109-122.

654 Dobin, A., Davis, C.A., Schlesinger, F., Drenkow, J., Zaleski, C., Jha, S., Batut, P., Chaisson, 655 M., and Gingeras, T.R. (2013). STAR: ultrafast universal RNASeq aligner. Bioinformatics 29, 656 15-21.

657 Dong, E., Du, H., and Gardner, L. (2020). An interactive web-based dashboard to track 658 COVID-19 in real time. Lancet Infect. Dis. 20, 533-534.

659 Duan, X., Ponomareva, L., Veeranki, S., \& Choubey, D. (2011). IFI16 induction by glucose re660 striction in human fibroblasts contributes to autophagy through activation of the 661 ATM/AMPK/p53 pathway. PloS one, 6(5), e19532. 662 https://doi.org/10.1371/journal.pone.0019532

663 Dyall, J., Coleman, C.M., Hart, B.J., Venkataraman, T., Holbrook, M.R., Kindrachuk, J., John664 son, R.F., Olinger, G.G., Jahrling, P.B., Laidlaw, M., et al. (2014). Repurposing of clinically de665 veloped drugs for treatment of Middle East respiratory syndrome coronavirus infection. 666 Antimicrob. Agents Chemother. 58, 4885-4893.

667 Ewers, H., and Helenius, A. (2011). Lipid-mediated endocytosis. Cold Spring Harb. Perspect. 668 Biol. 3, a004721.

669 Fabregat, A., Sidiropoulos, K., Garapati, P., Gillespie, M., Hausmann, K., Haw, R., Jassal, B., 670 Jupe, S., Korninger, F., McKay, S., et al. (2016). The Reactome pathway Knowledgebase. Nu671 cleic Acids Res. 44, D481-7. 
672 Fantini, J., Chahinian, H., Yahi, N. (2020). Leveraging coronavirus binding to gangliosides for 673 innovative vaccine and thera-peutic strategies against COVID-19. Biochem Biophys Res 674 Commun. 29, 132-136.

675 Fajnzylber, J., Regan, J., Coxen, K., Corry, H., Wong, C., Rosenthal, A., Worrall, D., Giguel, 676 F., Piechocka-Trocha, A., Atyeo, C., et al. (2020). SARS-CoV-2 viral load is associated with 677 increased disease severity and mortality. Nat. Commun. 11, 5493.

678 Gassen, N.C., Niemeyer, D., Muth, D., Corman, V.M., Martinelli, S., Gassen, A., Hafner, K., 679 Papies, J., Mösbauer, K., Zellner, A., et al. (2019). SKP2 attenuates autophagy through 680 Beclin1-ubiquitination and its inhibition reduces MERS-Coronavirus infection. Nat. Commun. $68110,5770$.

682 Gassen, N.C., Papies, J., Bajaj, T. et al. (2021) SARS-CoV-2-mediated dysregulation of me683 tabolism and autophagy uncovers host-targeting antivirals. Nat Commun 12, 3818

684 Gautret, P., Lagier, J.-C., Parola, P., Hoang, V.T., Meddeb, L., Mailhe, M., Doudier, B., 685 Courjon, J., Giordanengo, V., Vieira, V.E., et al. (2020). Hydroxychloroquine and azithromycin 686 as a treatment of COVID-19: results of an open-label non-randomized clinical trial. Int. J. 687 Antimicrob. Agents 56, 105949.

688 Ghaffari, S., Roshanravan, N., Tutunchi, H., Ostadrahimi, A., Pouraghaei, M., and Kafil, B. 689 (2020). Oleoylethanolamide, A Bioactive Lipid Amide, as A Promising Treatment Strategy for 690 Coronavirus/COVID-19. Arch Med Res 51, 464-467.

691 Goldman, D.L., Aldrich, M.L., Hagmann, S.H.F., Bamford, A., Camacho-Gonzalez, A., 692 Lapadula, G., Lee, P., Bonfanti, P., Carter, C.C., Zhao, Y., et al. (2021). Compassionate Use of 693 Remdesivir in Children With Severe COVID-19. Pediatrics 147, e2020047803.

694 Gordon, D.E., Jang, G.M., Bouhaddou, M., Xu, J., Obernier, K., White, K.M., O’Meara, M.J., 695 Rezelj, V.V., Guo, J.Z., Swaney, D.L., et al. (2020). A SARS-CoV-2 protein interaction map re696 veals targets for drug repurposing. Nature 583, 459-468.

697 Gorgas, K., Teigler, A., Komljenovic, D., and Just, W.W. (2006). The ether lipid-deficient 698 mouse: tracking down plasmalogen functions. Biochim. Biophys. Acta 1763, 1511-1526. 
699 Harris, M.A., Clark, J., Ireland, A., Lomax, J., Ashburner, M., Foulger, R., Eilbeck, K., Lewis, 700 S., Marshall, B., Mungall, C., et al. (2004). The Gene Ontology (GO) database and informatics 701 resource. Nucleic Acids Res. 32, D258-61.

702 Hwang J., Lee H.J., Lee W.H., Suk K. (2010). NF-kB as a common signaling pathway in 703 ganglioside-induced autophagic cell death and activation of astrocytes $\mathrm{J}$ Neuroimmunol. 704 226(1-2), 66-72.

705 Jackson, W.T. (2015). Viruses and the autophagy pathway. Virology 479-480, 450-456.

706 Jeon, S., Ko, M., Lee, J., Choi, I., Byun, S.Y., Park, S., Shum, D., and Kim, S. (2020). Identifi707 cation of Antiviral Drug Candidates against SARS-CoV-2 from FDA-Approved Drugs. 708 Antimicrob. Agents Chemother. 64 ,e00819-20.

709 Ji, W., Wang, W., Zhao, X., Zai, J., and Li, X. (2020). Cross-species transmission of the newly 710 identified coronavirus 2019-nCoV. J. Med. Virol. 92, 433-440.

711 Jiang Z, Wei F, Zhang Y, Wang T, Gao W, Yu S, Sun H, Pu J, Sun Y, Wang M, Tong Q, Gao 712 C, Chang KC, Liu J. (2021). IFI16 directly senses viral RNA and enhances RIG-I transcription 713 and activation to restrict influenza virus infection. Nat Microbiol 6, 932-945.

714 Kang, K.W., Kim, S., Cho, Y.-B., Ryu, S.R., Seo, Y.-J., and Lee, S.-M. (2019). Endogenous n-3 715 Polyunsaturated Fatty Acids Are Beneficial to Dampen CD8+ T Cell-Mediated Inflammatory 716 Response upon the Viral Infection in Mice. Int. J. Mol. Sci. 20, 4510.

717 Kao, J.C., HuangFu, W.C., Tsai, T.T., Ho, M.R., Jhan, M.K., Shen, T.J., Tseng, P.C., Wang, 718 Y.T., Lin, C.F. (2018). The antiparasitic drug niclosamide inhibits dengue virus infection by in719 terfering with endosomal acidification independent of mTOR. PLoS Negl Trop Dis. 12, 720 e0006715. doi:

721 Kim B, Arcos S, Rothamel K, Jian J, Rose KL, McDonald WH, Bian Y, Reasoner S, Barrows 722 NJ, Bradrick S, Garcia-Blanco MA, Ascano M. (2020). Discovery of Widespread Host Protein 723 Interactions with the Pre-replicated Genome of CHIKV Using VIR-CLASP. Mol Cell 78, 624724 640.e7. 
725 Kim, J.-J., Lee, H.-M., Shin, D.-M., Kim, W., Yuk, J.-M., Jin, H.S., Lee, S.-H., Cha, G.-H., Kim, 726 J.-M., Lee, Z.-W., et al. (2012). Host cell autophagy activated by antibiotics is required for their 727 effective antimycobacterial drug action. Cell Host Microbe 11, 457-468.

728 Koelmel, J.P., Kroeger, N.M., Ulmer, C.Z., Bowden, J.A., Patterson, R.E., Cochran, J.A., Bee729 cher, C.W.W., Garrett, T.J., and Yost, R.A. (2017). LipidMatch: an automated workflow for rule730 based lipid identification using untargeted high-resolution tandem mass spectrometry data. 731 BMC Bioinformatics 18, 331.

732 Koyuncu, E., Purdy, J.G., Rabinowitz, J.D., and Shenk, T. (2013). Saturated very long chain 733 fatty acids are required for the production of infectious human cytomegalovirus progeny. PLoS 734 Pathog. 9, e1003333.

735 Kuleshov, M.V., Jones, M.R., Rouillard, A.D., Fernandez, N.F., Duan, Q., Wang, Z., Koplev, S., 736 Jenkins, S.L., Jagodnik, K.M., Lachmann, A., et al. (2016). Enrichr: a comprehensive gene set 737 enrichment analysis web server 2016 update. Nucleic Acids Res. 44, W90-7.

738 Liao, Y., Smyth, G.K., and Shi, W. (2014). featureCounts: an efficient general purpose program 739 for assigning sequence reads to genomic features. Bioinformatics 30, 923-930.

740 Liberzon, A., Birger, C., Thorvaldsdóttir, H., Ghandi, M., Mesirov, J.P., and Tamayo, P. (2015). 741 The Molecular Signatures Database (MSigDB) hallmark gene set collection. Cell Syst. 1, 417742425.

743 Liu Y, Luo X, Shan H, Fu Y, Gu Q, Zheng X, Dai Q, Xia F, Zheng Z, Liu P, Yin XM, Hong L, Li 744 M. (2019). Niclosamide Triggers Non-Canonical LC3 Lipidation. Cells 2019 8, 248.

745 Liu, J., Cao, R., Xu, M., Wang, X., Zhang, H., Hu, H., Li, Y., Hu, Z., Zhong, W., and Wang, M. 746 (2020). Hydroxychloroquine, a less toxic derivative of chloroquine, is effective in inhibiting 747 SARS-CoV-2 infection in vitro. Cell Discov. 6, 16.

748 Love, M.I., Huber, W., and Anders, S. (2014). Moderated estimation of fold change and' ' dis749 persion for RNASeq data with DESeq2. Genome Biol. 15, 550. 
750 Luquain-Costaz, C., Rabia, M., Hullin-Matsuda, F., and Delton, I. (2020). 751 Bis(monoacylglycero)phosphate, an important actor in the host endocytic machinery hijacked 752 by SARS-CoV-2 and related viruses. Biochimie 179, 247-256.

753 Mahmud, I., and Garrett, T.J. (2020). Mass Spectrometry Techniques in Emerging Pathogens 754 Studies: COVID-19 Perspectives. J Am Soc Mass Spectrom 31, 2013-2024.

755 Marsh, M., and Helenius, A. (2006). Virus entry: open sesame. Cell 124, 729-740.

756 Martin, M. (2011). Cutadapt removes adapter sequences from high-throughput sequencing 757 reads. EMBnet j. 17, 10.

758 Mauthe, M., \& Reggiori, F. (2016). ATG proteins: Are we always looking at autopha759 gy?. Autophagy, 12(12), 2502-2503. https://doi.org/10.1080/15548627.2016.1236878

760 Mauthe, M., Orhon, I., Rocchi, C., Zhou, X., Luhr, M., Hijlkema, K.-J., Coppes, R.P., Engedal, 761 N., Mari, M., and Reggiori, F. (2018). Chloroquine inhibits autophagic flux by decreasing 762 autophagosome-lysosome fusion. Autophagy 14, 1435-1455.

763 Mazzon, M., and Mercer, J. (2014). Lipid interactions during virus entry and infection. Cell 764 Microbiol. 16, 1493-1502.

765 Miyanari, Y., Atsuzawa, K., Usuda, N., Watashi, K., Hishiki, T., Zayas, M., Bartenschlager, R., 766 Wakita, T., Hijikata, M., and Shimotohno, K. (2007). The lipid droplet is an important organelle 767 for hepatitis C virus production. Nat. Cell Biol. 9, 1089-1097.

768 Nguyen, A., Guedán, A., Mousnier, A., Swieboda, D., Zhang, Q., Horkai, D., Le Novere, N., 769 Solari, R., and Wakelam, M.J.O. (2018). Host lipidome analysis during rhinovirus replication in 770 HBECs identifies potential therapeutic targets. J. Lipid Res. 59, 1671-1684.

771 O'Leary, N.A., Wright, M.W., Brister, J.R., Ciufo, S., Haddad, D., McVeigh, R., Rajput, B., 772 Robbertse, B., Smith-White, B., Ako-Adjei, D., et al. (2016). Reference sequence (RefSeq) da773 tabase at NCBI: current status, taxonomic expansion, and functional annotation. Nucleic Acids 774 Res. 44, D733-45. 
775 Ogata, H., Goto, S., Sato, K., Fujibuchi, W., Bono, H., and Kanehisa, M. (1999). KEGG: kyoto 776 encyclopedia of genes and genomes. Nucleic Acids Res. 27, 29-34.

777 Overmyer, K.A., Shishkova, E., Miller, I.J., Balnis, J., Bernstein, M.N., Peters-Clarke, T.M., 778 Meyer, J.G., Quan, Q., Muehlbauer, L.K., Trujillo, E.A., et al. (2021). Large-Scale Multi-omic 779 Analysis of COVID-19 Severity. Cell Syst. 12, 23-40.e7.

780 Pacha, O., Sallman, M.A., and Evans, S.E. (2020). COVID-19: a case for inhibiting IL-17? Nat. 781 Rev. Immunol. 20, 345-346.

782 Pang, Z., Chong, J., Zhou, G., Morais D., Chang, L., Barrette, M., Gauthier, C., Jacques, PE., 783 Li, S., and Xia, J. (2021) MetaboAnalyst 5.0: narrowing the gap between raw spectra and func784 tional insights Nucl. Acids Res. (doi: 10.1093/nar/gkab382)

785 Pindiprolu, S.K.S.S., and Pindiprolu, S.H. (2020). Plausible mechanisms of Niclosamide as an 786 antiviral agent against COVID-19. Med. Hypotheses 140, 109765.

787 Rizvi, Z.A., Dalal, R., Sadhu, S., Kumar, Y., Shrivastava, T., Gupta, S.K., Agarwal, S., Tripathy, 788 M.R., Yadav, A.K., Medigeshi, G.R., et al. (2021). Immunological and cardio-vascular patholo789 gies associated with SARS-CoV-2 infection in golden syrian hamster. BioRxiv.

790 Rubinsztein, D.C., Codogno, P., and Levine, B. (2012). Autophagy modulation as a potential 791 therapeutic target for diverse diseases. Nat. Rev. Drug Discov. 11, 709-730.

792 Salcher, S., Hermann, M., Kiechl-Kohlendorfer, U., Ausserlechner, M.J., Obexer, P. (2017) 793 C10ORF10/DEPP-mediated ROS accu-mulation is a critical modulator of FOXO3-induced au794 tophagy. Mol Cancer. 16, 95.

795 Sharma, A., Garcia Jr., G., Wang, Y., Plummer, J. T., Morizono, K., Arumugaswami, V., and 796 Svendsen, C. N. (2020). Human iPSC-Derived Cardiomyocytes Are Susceptible to SARS797 CoV-2 Infection. Cell Rep Med. 1, 100052.

798 Skotland, T., Iversen, T.G., Torgersen, M.L., and Sandvig, K. (2015). Cell-penetrating pep799 tides: possibilities and challenges for drug delivery in vitro and in vivo. Molecules 20, 1331380013323. 
801 Soudani, N., Hage-Sleiman, R., Karam, W., Dbaibo, G., and Zaraket, H. (2019). Ceramide 802 suppresses influenza A virus replication in vitro. J. Virol. 93.

803 Srivastava, R., Daulatabad, S. V., Srivastava, M., \& Janga, S. C. (2020). Role of SARS-CoV-2 804 in Altering the RNA-Binding Protein and miRNA-Directed Post-Transcriptional Regulatory Net805 works in Humans. International journal of molecular sciences, 21(19), 7090. 806 https://doi.org/10.3390/ijms21197090

807 Stukalov, A., Girault, V., Grass, V., Karayel, O., Bergant, V., Urban, C., Haas, D.A., Huang, Y., 808 Oubraham, L., Wang, A., et al. (2021). Multilevel proteomics reveals host perturbations by SARS-CoV-2 and SARS-CoV. Nature.

810 Tam, V.C., Quehenberger, O., Oshansky, C.M., Suen, R., Armando, A.M., Treuting, P.M., 811 Thomas, P.G., Dennis, E.A., and Aderem, A. (2013). Lipidomic profiling of influenza infection 812 identifies mediators that induce and resolve inflammation. Cell 154, 213-227.

813 Thai, T.P., Rodemer, C., Jauch, A., Hunziker, A., Moser, A., Gorgas, K., and Just, W.W. 814 (2001). Impaired membrane traffic in defective ether lipid biosynthesis. Hum. Mol. Genet. 10, 815 127-136.

816 Ulmer C.Z., Yost R.A., and Garrett T.J. (2017) Global UHPLC/HRMS Lipidomics Workflow for 817 the Analysis of Lymphocyte Suspension Cultures. In: Wood P. (eds) Lipidomics. 818 Neuromethods, vol 125. Humana Press, New York, NY. https://doi.org/10.1007/978-1-4939819 6946-3_13

820 V'kovski, P., Kratzel, A., Steiner, S., Stalder, H., and Thiel, V. (2020). Coronavirus biology and 821 replication: implications for SARS-CoV-2. Nat. Rev. Microbiol. 19, 155-170.

822 Vincent, M.J., Bergeron, E., Benjannet, S., Erickson, B.R., Rollin, P.E., Ksiazek, T.G., Seidah, 823 N.G., and Nichol, S.T. (2005). Chloroquine is a potent inhibitor of SARS coronavirus infection 824 and spread. Virol. J. 2, 69.

825 Weisberg, E., Parent, A., Yang, P. L., Sattler, M., Liu, Q., Liu, Q., Wang, J., Meng, C., 826 Buhrlage, S. J., Gray, N., \& Griffin, J. D. (2020). Repurposing of Kinase Inhibitors for Treat- 
827 ment of COVID-19. Pharmaceutical research, 37(9), 167. https://doi.org/10.1007/s11095-020$828 \quad 02851-7$

829 Wang, L., Wang, S., and Li, W. (2012). RSeQC: quality control of RNASeq experiments. Bioin830 formatics 28, 2184-2185.

831 Wang, M., Cao, R., Zhang, L., Yang, X., Liu, J., Xu, M., Shi, Z., Hu, Z., Zhong, W., and Xiao, 832 G. (2020). Remdesivir and chloroquine effectively inhibit the recently emerged novel corona833 virus (2019-nCoV) in vitro. Cell Res. 30, 269-271.

834 Wichit, S., Hamel, R., Yainoy, S., Gumpangseth, N., Panich, S., Phuadraksa, T., Saetear, P., 835 Monteil, A., Morales Vargas, R., \& Missé, D. (2019). Interferon-inducible protein (IFI) 16 regu836 lates Chikungunya and Zika virus infection in human skin fibroblasts. EXCLI journal, 18, 467837 476. https://doi.org/10.17179/excli2019-1271

838 Wu, C.-J., Jan, J.-T., Chen, C.-M., Hsieh, H.-P., Hwang, D.-R., Liu, H.-W., Liu, C.-Y., Huang, 839 H.-W., Chen, S.-C., Hong, C.-F., et al. (2004). Inhibition of severe acute respiratory syndrome coronavirus replication by niclosamide. Antimicrob. Agents Chemother. 48, 2693-2696.

841 Wyler, E., Mösbauer, K., Franke, V., Diag, A., Gottula, L.T., Arsiè, R., Klironomos, F., 842 Koppstein, D., Hönzke, K., Ayoub, S., Buccitelli, C., Hoffmann, K., Richter, A., Legnini, I., 843 Ivanov, A., Mari, T., Del Giudice, S., Papies, J., Praktiknjo, S., Meyer, T.F., Müller, M.A., Nie844 meyer, D., Hocke, A., Selbach, M., Akalin, A., Rajewsky, N., Drosten, C., and Landthaler, M. 845 (2021). Transcriptomic profiling of SARS-CoV-2 infected human cell lines identifies HSP90 as 846 target for COVID-19 therapy. iScience. 24, 102151.

847 Xie, Y., Li, J., Kang, R., and Tang, D. (2020). Interplay between lipid metabolism and autopha848 gy. Front. Cell Dev. Biol. 8, 431.

849 Xu, J., Shi, P.-Y., Li, H., and Zhou, J. (2020). Broad spectrum antiviral agent niclosamide and 850 its therapeutic potential. ACS Infect. Dis. 6, 909-915.

851 Yates, A.D., Achuthan, P., Akanni, W., Allen, J., Allen, J., Alvarez-Jarreta, J., Amode, M.R., 852 Armean, I.M., Azov, A.G., Bennett, R., et al. (2020). Ensembl 2020. Nucleic Acids Res. 48, 853 D682-D688. 
854 Young, M.M., Kester, M., and Wang, H.-G. (2013). Sphingolipids: regulators of crosstalk be855 tween apoptosis and autophagy. J. Lipid Res. 54, 5-19.

856 Yoshikawa, T., Hill, T.E., Yoshikawa, N., Popov, V.L., Galindo, C.L., Garner, H.R., Peters, C.J., 857 and Kent Tseng, C. (2010). Dynamic innate immune responses of human bronchial epithelial 858 cells to severe acute respiratory syndrome-associated coronavirus infection. PLoS One. 5, 859 e8729.

860 Zachari, M., \& Ganley, I. G. (2017). The mammalian ULK1 complex and autophagy initia861 tion. Essays in biochemistry, 61(6), 585-596. https://doi.org/10.1042/EBC20170021

862 Zamaraev AV, Zhivotovsky B, Kopeina GS. Viral Infections: Negative Regulators of Apoptosis 863 and Oncogenic Factors. Biochemistry (Mosc). 2020 Oct;85(10):1191-1201. doi: 864 10.1134/S0006297920100077. PMID: 33202204; PMCID: PMC7590567.

865 Zhou, Y., Hou, Y., Shen, J., Huang, Y., Martin, W., and Cheng, F. (2020). Network-based drug 866 repurposing for novel coronavirus 2019-nCoV/SARS-CoV-2. Cell Discov. 6, 14.

867 Zhu, Y., Feng, .F, Hu, G., Wan,g Y., Yu, Y., Zhu, Y., Xu, W., Cai, X., Sun, Z., Han, W., Ye, R., 868 Qu, D., Ding, Q., Huang, X., Chen, H., Xu, W., Xie, Y., Cai, Q., Yuan, Z., Zhang, R. (2021). A 869 genome-wide CRISPR screen identifies host factors that regulate SARS-CoV-2 entry. Nat 870 Commun. 12, 961.

\section{FIGURE LEGENDS}

873 Figure 1. Workflow for lipidomic and transcriptomic profiling of Vero E6 cells after 874 SARS-CoV-2 infection with and without niclosamide treatment. We used a time-of875 addition assay experimental design to (i) capture the lipidomic profile of SARS-CoV-2 infected 876 Vero E6 cells, and (ii) explore the effect of niclosamide on the lipidomic profile of Vero E6 cells 877 when added in the absence of infection, with SARS-CoV-2 virus, or at 24h post-infection. Each 878 replicate experimental condition $(n=3)$ was processed for LC-HRMS/MS or RNASeq analyses. 879 Samples were seeded $48 \mathrm{~h}$ prior to the start of the experiment $(\mathrm{t}=\mathrm{0h})$. For all samples, media 
880 was changed at the start of the experiment $(\mathrm{t}=\mathrm{Oh})$ and infected with virus (for infected sample

881 groups). Sample collection is denoted by an up arrow and tube above the timeline, media

882 changes are denoted by red bottles, addition of virus and DMSO/drug are denoted as well.

883 Separate samples for each condition were collected for LC-HRMS/MS and RNAseq analysis,

884 respectively.

885

Figure 2. Global lipidomics analysis in SARS-CoV-2 infected Vero E6 cells at $16 \mathrm{~h}$ and

48h. (A) Hierarchical cluster heatmap analysis depicting the major affected lipid clustering between $16 \mathrm{~h}$ and $48 \mathrm{~h}$ infection. (B) Volcano plot showing the differential lipid abundance with SARS-CoV-2 infection between $16 \mathrm{~h}$ and $48 \mathrm{~h}$. The primary significant upregulated lipids were 890 ether-linked (plasmanyl and plasmenyl). (C) Bar graph showing the total abundance of ether 891 lipids between the time points. (D) Extracted ion chromatogram comparison showing relative 892 peak intensity of a single ether lipid between $16 \mathrm{~h}$ (blue) and $48 \mathrm{~h}$ (magenta) infection. The ar893 row points to the lipid identified as Plasmanyl-PC $(\mathrm{O}-18: 0 / 16: 0)+\mathrm{H}$ at retention time 9.16. Iden894 tification was confirmed by accurate mass and MS/MS.

Figure 3. Comparison of individual lipid classes at early (16h) and late (48h) viral infec-

897 tion. FDR P-value based on T-test shown above each plot. Individual triangles represent 898 each lipid detected and expression compared at both time points. Abbreviations: TG-

899 triglycerides, DG-diglycerides, CL-cardiolipin, CE-cholesterol ester, SM-sphingomyelin, PC-

900 phosphatidylcholine, PE-phosphatidylethanolamine, PS-phosphatidylserine, PI-

901 phosphatidylinositol, PG-phosphatidylglycerol, LPC-lysophosphatidylcholine, LPE- 
902 lysophosphatidylethanolamine, oxLPC-oxidized lysophosphatidylcholine, HexCer-

903 hexosylceramide, CerNS-ceramide with non-hydroxy fatty acid, n.s.-not significant.

Figure 4. Niclosamide modulates lipid metabolism in Vero E6 cells in the absence of

906 SARS-CoV-2 infection. Heatmap showing the changes in neutral lipids across the time points 907 with and without NIC (A). Bar graphs of the significant lipids at $16 \mathrm{~h}$ vs $48 \mathrm{~h}$ (B) and $48 \mathrm{~h}$ vs $48 \mathrm{~h}$ $908+\mathrm{NIC}(\mathrm{C})$. Fold change of less than 1.5 and $p$-value less than or equal to 0.05 .

911 at $48 \mathrm{~h}$. (A) Heatmap of the top 70 lipids (Fold change $\geq 1.5$ and $p$-value $\leq 0.05$ ). (B) Volcano

912 plot showing the significant up and down regulated lipids with NIC at 48h. (C) The total ether 913 lipids were down regulated with NIC treatment.

915 Figure 6. Significantly expressed genes across SARS-CoV-2 and Niclosamide condi-

916 tions. Genes were designated as significantly expressed using DESeq2 when their Bonferroni

917 adjusted P-value was $\leq 0.05$, and the $\mid$ log2(Fold Change) | was $\geq 1$. Each dot represents an indi918 vidual gene. Blue dots are significantly downregulated genes, red dots are significantly

919 upregulated genes, and grey dots are not significantly differentially expressed genes. (A)

920 DMSO treated no virus versus DMSO treated, virus samples at 16 hours, (B) DMSO treated no 921 virus versus DMSO treated virus samples at 48 hours, (C) Niclosamide treated no virus versus 922 niclosamide treated virus samples at 16 hours, (D) Niclosamide treated no virus versus 923 niclosamide treated virus samples at 48 hours, (E) DMSO treated no virus versus niclosamide 
924 treated no virus samples at 16 hours, $(F)$ DMSO treated no virus versus niclosamide treated 925 no virus samples at 48 hours, (G) DMSO treated virus versus niclosamide treated virus sam926 ples at 16 hours, (H) DMSO treated virus at 16 hours versus 48 hours, (I) Niclosamide treated, 927 no virus samples at 16 vs 48 hours.

928

929 Figure 7. The effect of SARS-CoV-2 infection and NIC treatment on host cell lipid me-

930 tabolism. SARS-CoV-2 infection in Vero E6 cells alters host cell lipid metabolism during early

931 and late stages of infection. Increased transcription of lipid receptors LRP2 and VLDLR, and 932 phosphorylation signaling regulators are observed throughout viral infection. Changes in TG 933 composition from unsaturated to saturated acyl-chains occurs as a function of viral replication, 934 with an overall decrease in TG lipids at late infection timepoints. This change corresponds with 935 an increase to DG and BMP lipids that is indicative of energy consumption and incorporation 936 into membranes and vesicles, activation of autophagy pathways, as well as impacting viral rep937 lication. Treatment of cells with NIC alters lipid composition and gene regulation corresponding 938 to apoptosis and autophagy related pathways. Decreases to ether lipids (TGs and DGs) and 939 BMP are observed and reflect a decrease to exocytosis pathways for viral egress. 
Time-of-Addition Assay

Co-inoculation with treatment

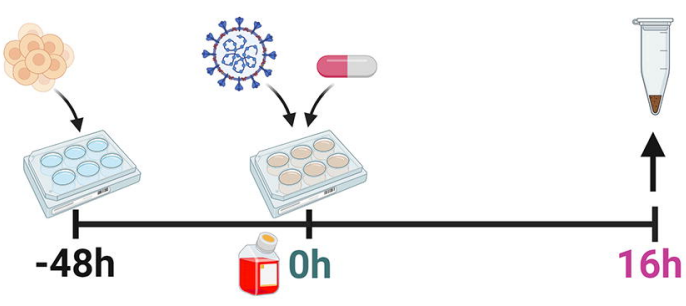

Treatment post-inoculation

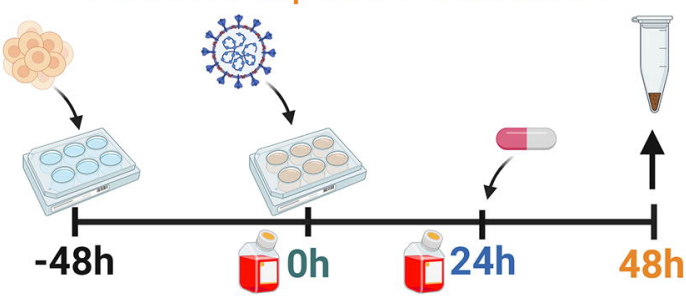

$16 \mathrm{~h}$

$16 \mathrm{~h} 7 \quad$ Data Acquisition
LC-HRMS/MS

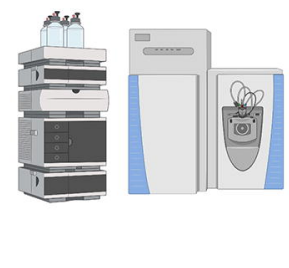

Expression \& Pathway Analyses

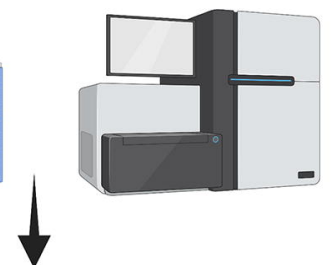

$48 \mathrm{~h}$

$\sqrt{2} \sqrt{\circ}$

๔

$48 \mathrm{~h}$

$\sqrt{7} \sqrt{7}$ $\checkmark$.

$\theta$ 幥察

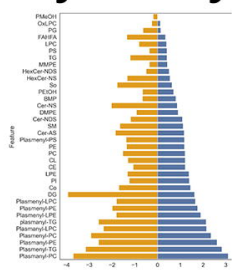

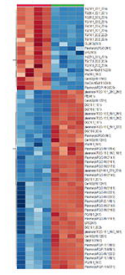




\section{B $\quad 48 \mathrm{~h}$ SARS-CoV-2/16h SARS-CoV-2}

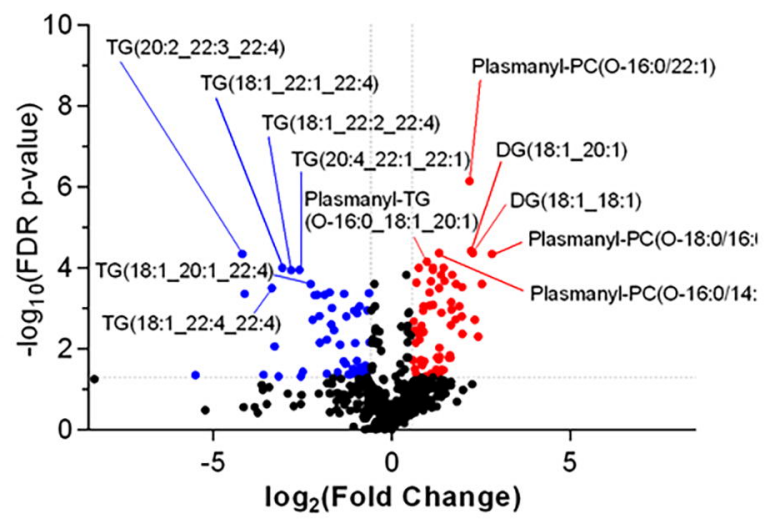

D

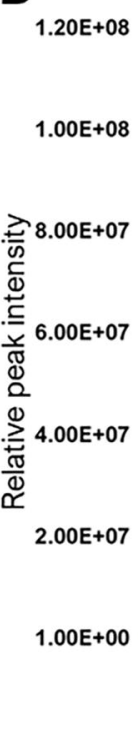

\section{ETHER LIPIDS}

TG(18:1_22:1_22:4) TG(20:4_22:1_22:1) TG(20:2_22:3_22:4) TG(18:1_22:2_22:4) TG(18:1_20:1_22:4) TG(18:1_20:2 22:2) TG(18:1_20:2_22:4) $\mathrm{CL}(36: 3)(36: 3)$

Plasmanyl-LPC(O-20:1) $\operatorname{LPC}(16: 0)$

TG(22:3_22:3_22:3)

TG(16:0_20:2 25:0)

HexCer.NS(d18:1/22:0)

PG(16:0_16:0)

Cer-NS(d18:1/16:0)

HexCer.NS(d18:1/24:0)

Plasmenyl.PE(P-16:0/20:5)

plasmanyl.TG(O-16:1_20:0_20:0)

PE(44:1)

Cer-NS(d18:1/22:1)

DG(18:1_18:1)

plasmanyl.TG(0.16:0_18:1_20:1) plasmanyl.TG(O.16:0_18:1_20:2 DG(16:1_18:1)

plasmanyl.TG(O-16:0_18:1_22:2) DG(18:0_20:4)

Plasmanyl-LPC (0.16:0)

Cer-NS(d18:1/24:0)

PG(16:1_18:1)

Plasmanyl-PC(O-18:1/20:4)

plasmanyl.TG(O-18:0_16:0_18:1)

Plasmanyl.PC $(0.16: 0 / 14: 1)$

Plasmanyl.PC(O-16:0/16:1)

Plasmanyl-PC(O-16:0/17:0)

plasmenyl-TG(P-16:1_22:0_22:0)

PlasmanylPC(0.16-0/14:0)

DG(18:1_20:1)

Cer-NS(d18:1/24:1)

Plasmanyl.PC(0.16:0/18:1)

Plasmanyl.PC $(0.16: 0 / 22: 1)$

Plasmanyl.PC(O-16:0/22.2)

Plasmenyl.PC(P.20:1/18:1)

Plasmanyl-PC(O-16:0/14:0)

Plasmanyl.PC(O-18:0/16:0)

PC(18:1_24:1)

Plasmanyl-PC(O.18:1/16:1)

$\operatorname{LPC}(24: 1)$

DG(18:1_20:2)

plasmanyl.TG(O.18:1_16:0_18:1)

Cer-NS(d18:1/25:1)

SM(d 18:2/16:0)

Plasmenyl-PC(P.16:1/18:0)

Plasmenyl.PC(P-18:0/18:1)

Plasmanyl.PC(O.18:1/22:2)

Plasmenyl-PC(P-16:1/18:1)

$\mathrm{PC}(16: 1$ 18:2)

Plasmenyl.PC(P.16:0/22:1)

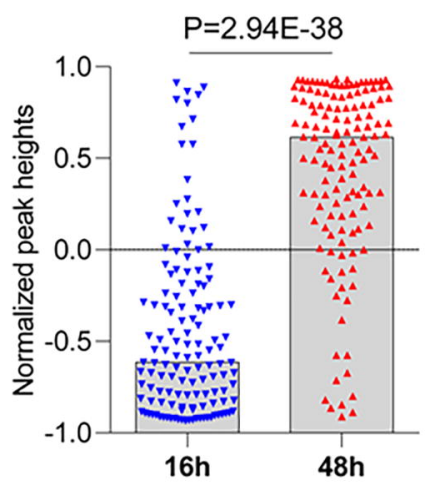

48h SARS-CoV-2

16h SARS-CoV-2 

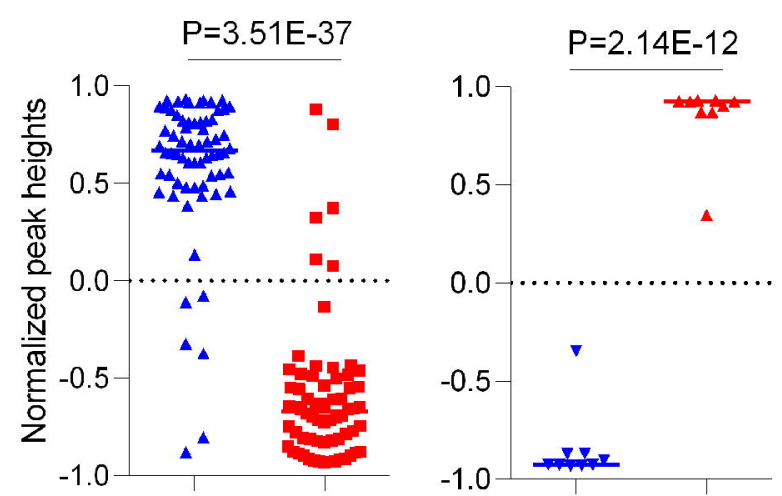

n.s.
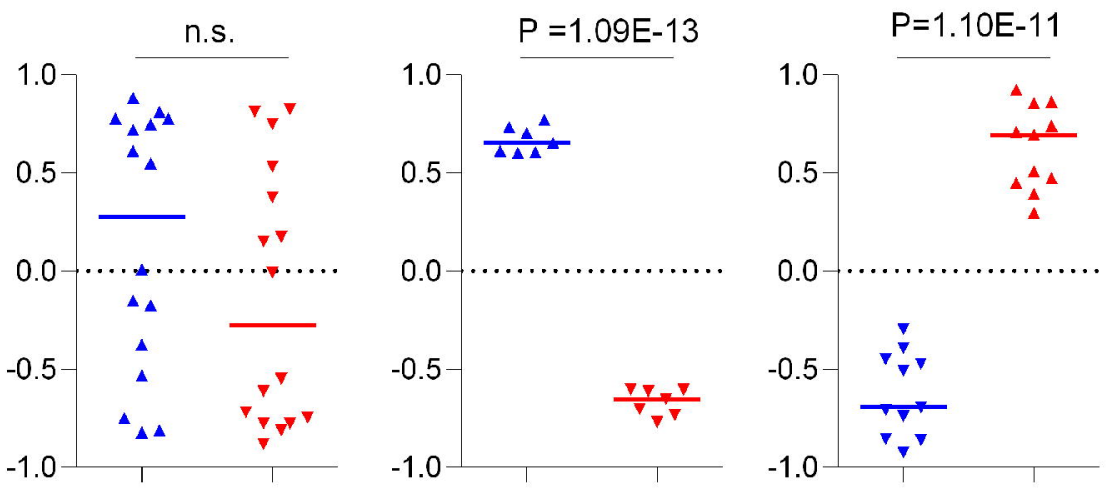

\section{PC}

PE

PS

PI
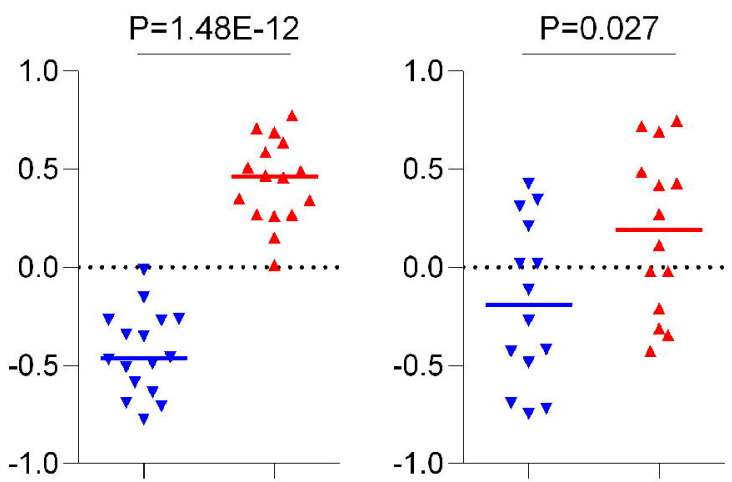

PG

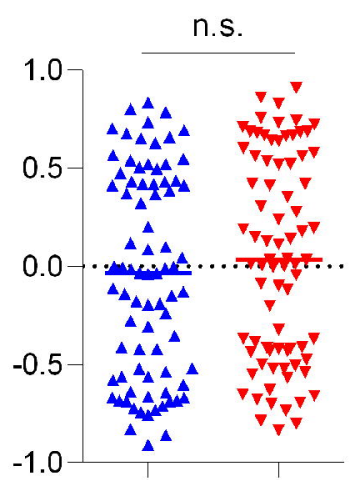

LPC

LPE

OXLPC
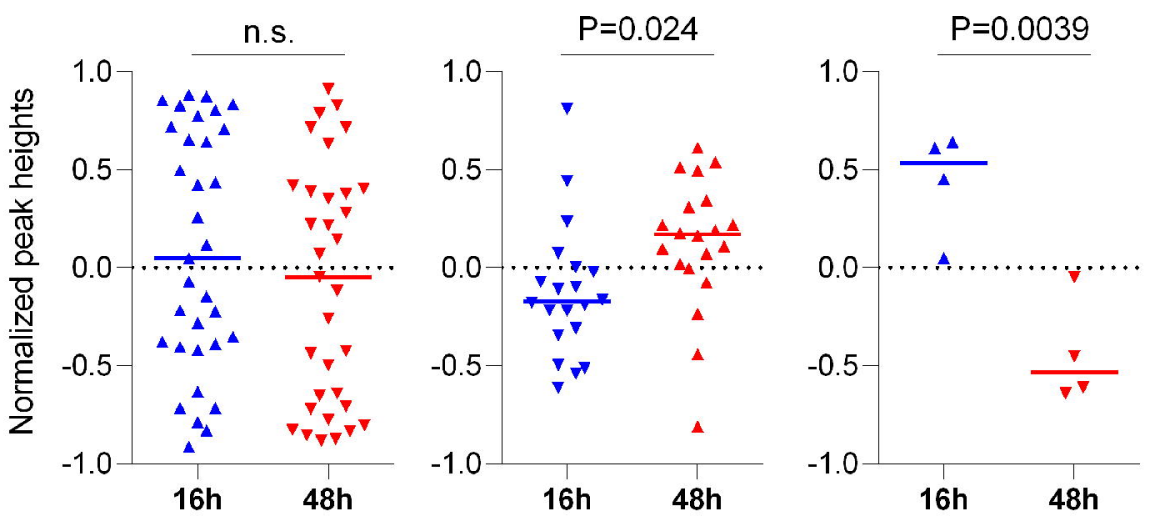

\section{HexCer}

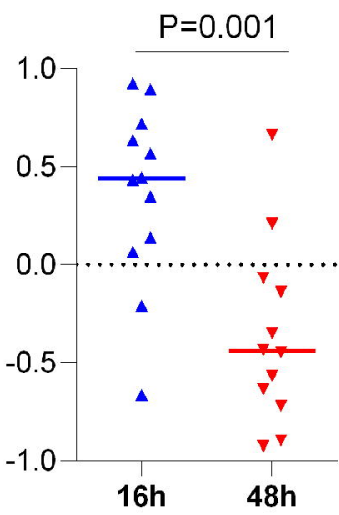

\section{CerNS}

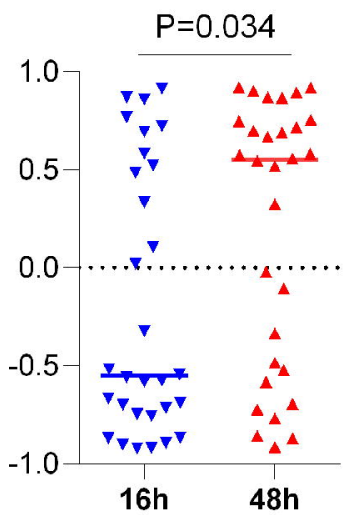




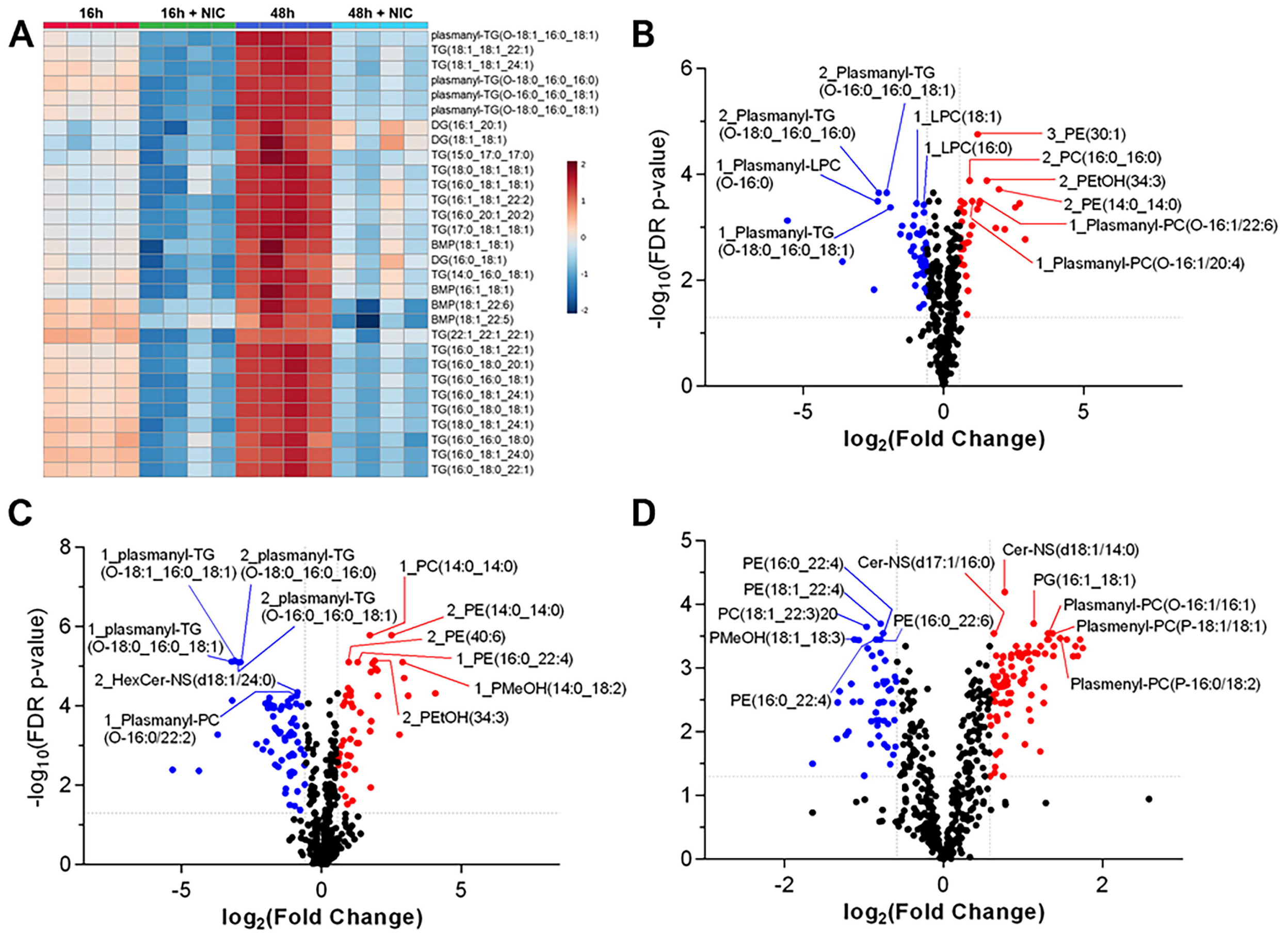


A SARS-CoV-2 SARS-CoV-2 + NIC

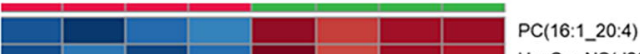

HexCer-NS(d20:1/15:0)

PC(16:1_20:4)

$\mathrm{PC}\left(13: 0 \_20: 3\right)$

$\mathrm{PE}(42: 10)$

PC(14:0_14:1)

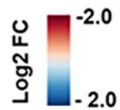

$\operatorname{PE}\left(20: 4 \_20: 4\right) \cdot H$

HexCer-NDS(d18:0/14:0)

$\operatorname{PE}(30: 1)$

PEtOH(40:9)

PC(16:1_22:6)

Plasmenyl-PE(P-16:0/20:5)

$P C\left(18: 2 \_20: 4\right)$

PC(18:1_20:5)

PC(16:1_22:6)

$\operatorname{LPC}(14: 1)$

SM(d18:1/14:0)

plasmenyl-TG(P-16:1_22:0_22:0)

Plasmanyl-PC(O-18:1/22:2)

PC(18:1_20:1)

Plasmanyl-PC(O-18:1/20:4)

DG(16:0_16:1)

Plasmanyl-PC(O-16:0/16:1)

Plasmanyl-PC(O-16:0/17:0)

Plasmanyl-PC(O-16:0/18:1)

Plasmenyl-PC(P-18:0/18:1)

Plasmanyl-PC(O-16:0/22:1)

PG(16:1_18:1)

plasmanyl-TG(0-18:1_16:0_18:1) LPC(24:1)

Plasmenyl-PC(P-18:0/18:1)

HexCer-NS(d18:1/14:0)

HexCer-NS(d18:1/18:0)

plasmanyl-TG(0-16:0_16:0_18:1)

plasmanyl-TG(0-16:0_18:1_20:1)

Plasmanyl-PC(O-18:1/20:4)

plasmanyl-TG(0-18:0_16:0_18:1)

plasmanyl-TG(0-18:0_16:0_20:1)

Plasmanyl-PC(O-16:0/22:4)

plasmanyl-TG(0-16:0_18:1_20:2)

plasmanyl-TG(0-16:0_18:1_22:2)

plasmanyl-TG(0-16:0_18:1_22:6)

plasmanyl-TG(0-16:1_20:0_20:0)

Cer-NS(d18:1/22:0)

Cer-NS(d18:1/22:1)

Cer-NS(d18:1/23:0)

Cer-NS(d18:1/24:0)

Cer-NS(d18:1/24:0)

plasmanyl-TG(0-18:1_16:0_16:1)

plasmanyl-TG(O-18:1_16:0_22:1)

plasmanyl-TG(0-20:0_20:2_20:2)

plasmanyl-TG(0-18:0_18:1_22:1)

PG(17:1_17:1)

plasmanyl-TG(0-18:0_16:0_22:1)

DG(18:0_20:4)

DG(18:1_18:1)

plasmanyl-TG(0-18:0_18:1_22:4)

DG(16:1_18:1)

Plasmanyl-PC(O-18:0/16:0)

Cer-NS(d18:2/24:1)

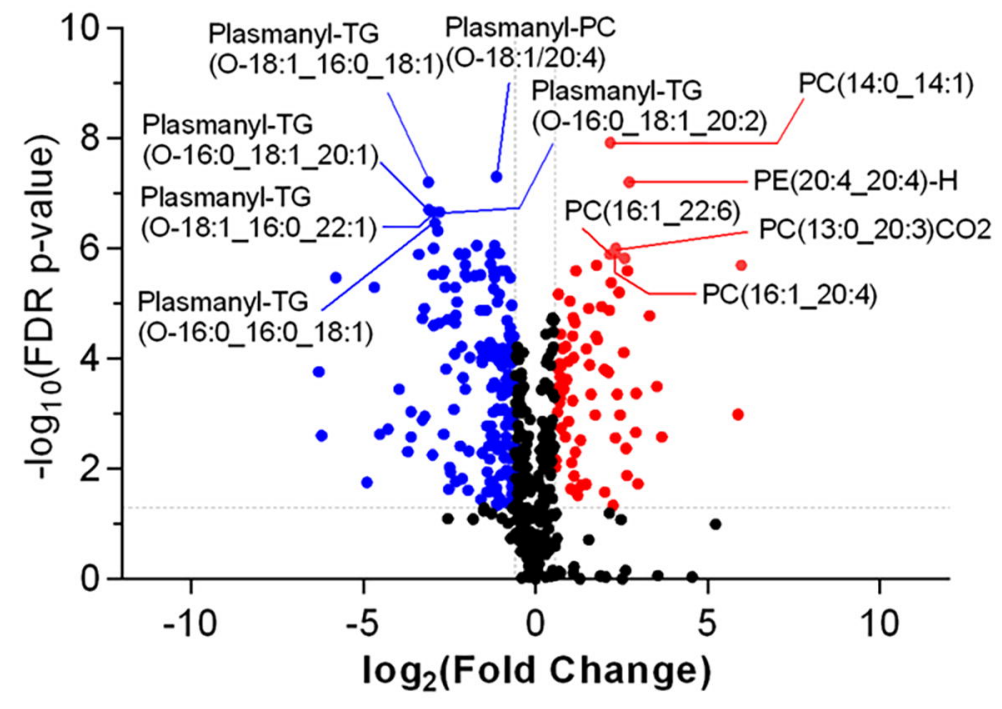

C

ETHER LIPIDS

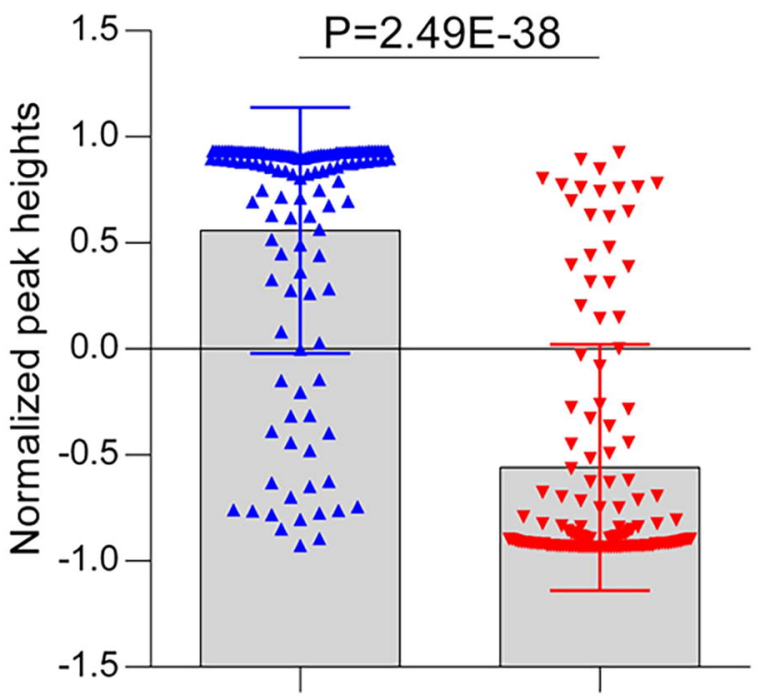

SARS-CoV-2 SARS-CoV-2+NIC 


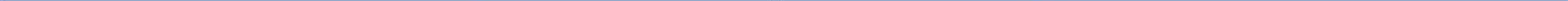

